\title{
Where is the Fluoro Wall? - A Quantum Chemical Investigation
}

Julian D. Rolfes, Maurice van Gastel, Frank Neese

Supporting Information 


\section{Abbreviations}

An incomplete list of the technical abbreviations most frequently used in this work is provided to facilitate reading for the general readership:

AILFT: $a b$ initio ligand field theory

AOM: angular overlap model

NPA: natural population analysis

QRO: quasi-restricted orbitals

\section{Model Complex}

\subsection{Spin State Analysis}

Table S1: Multiplicities of the ground states (GS) as well as the $2^{\text {nd }}$ and $3^{\text {rd }}$ most stable spin states with relative energy differences in $\mathrm{kJ} / \mathrm{mol}$ of $\mathrm{M}-\mathrm{X}$ complexes with $\mathrm{X}=\mathrm{F}$ and $\mathrm{X}=\mathrm{O}$.

\begin{tabular}{|c|c|c|c|c|c|c|c|c|c|c|}
\hline \multirow[b]{2}{*}{$M=$} & \multicolumn{5}{|c|}{$X=F$} & \multirow[b]{2}{*}{ GS } & \multicolumn{2}{|c|}{$x=0$} & \multirow[b]{2}{*}{$3^{\text {rd }}$} & \multirow[b]{2}{*}{$\begin{array}{l}3^{\mathrm{rd}}-\mathrm{GS} \\
{[\mathrm{kJ} / \mathrm{mol}]}\end{array}$} \\
\hline & GS & $2^{\text {nd }}$ & $\begin{array}{l}2^{\text {nd }}-\mathrm{GS} \\
{[\mathrm{kJ} / \mathrm{mol}]}\end{array}$ & $3^{\text {rd }}$ & $\begin{array}{l}3^{\text {rd }}-\mathrm{GS} \\
{[\mathrm{kJ} / \mathrm{mol}]}\end{array}$ & & $2^{\text {nd }}$ & $\begin{array}{l}2^{\text {nd }}-\mathrm{GS} \\
{[\mathrm{kJ} / \mathrm{mol}]}\end{array}$ & & \\
\hline $\mathrm{Mn}$ & 4 & 2 & 99.3 & - & - & 4 & 2 & 17.5 & - & - \\
\hline $\mathrm{Fe}$ & 3 & 5 & 119.4 & 1 & 148.8 & 3 & 5 & 81.4 & 1 & 127.1 \\
\hline Co & 2 & 6 & 104.9 & 4 & 128.5 & 2 & 4 & 53.5 & 6 & 187.6 \\
\hline $\mathrm{Ni}$ & 1 & 3 & 77.0 & - & - & 3 & 1 & 63.5 & 5 & 78.9 \\
\hline Tc & 4 & 2 & 12.1 & - & - & 2 & 4 & 96.6 & - & - \\
\hline $\mathrm{Ru}$ & 3 & 1 & 73.9 & 5 & 332.0 & 3 & 1 & 89.6 & 5 & 238.8 \\
\hline $\mathrm{Rh}$ & 2 & - & - & - & - & 2 & 4 & 119.6 & 6 & 434.5 \\
\hline $\mathrm{Pd}$ & 1 & 3 & 227.0 & - & - & 1 & 3 & 12.3 & - & - \\
\hline $\mathrm{Re}$ & 4 & 2 & 14.5 & - & - & 2 & 4 & 121.9 & - & - \\
\hline Os & 3 & 1 & 76.4 & 5 & 311.1 & 3 & 1 & 65.2 & 5 & 251.3 \\
\hline Ir & 2 & 4 & 301.5 & 6 & 662.7 & 2 & 4 & 149.6 & 6 & 481.5 \\
\hline $\mathrm{Pt}$ & 1 & 3 & 336.4 & 5 & 684.8 & 1 & 3 & 107.4 & - & - \\
\hline
\end{tabular}

\subsection{Geometries}

The DFT optimized geometries of all 24 complex ground states are given below.

$\begin{array}{lrrr}\text { Mn-F: } & & \\ \mathrm{Mn} & 0.00000 & 0.00000 & 0.00000 \\ \mathrm{~F} & 0.00000 & 0.00000 & 1.76884 \\ \mathrm{O} & -1.46486 & -0.03069 & -3.61213 \\ \mathrm{O} & -0.11106 & 0.01187 & -1.84653 \\ \mathrm{~N} & 0.00277 & -1.83801 & -0.08709 \\ \mathrm{~N} & 1.94797 & -0.00000 & -0.20167 \\ \mathrm{~N} & -1.97104 & -0.00401 & -0.03652 \\ \mathrm{~N} & -0.02404 & 1.83638 & -0.03707 \\ \mathrm{C} & -2.45660 & 0.01142 & -1.43979 \\ \mathrm{C} & -1.30396 & -0.00658 & -2.43595 \\ \mathrm{C} & 2.16900 & -1.29719 & -0.97647\end{array}$




$\begin{array}{lr}\mathrm{C} & 1.10288 \\ \mathrm{C} & 2.17396 \\ \mathrm{C} & -1.19394 \\ \mathrm{C} & -2.22334 \\ \mathrm{C} & 1.10601 \\ \mathrm{C} & -2.24829 \\ \mathrm{C} & -1.20982 \\ \mathrm{C} & 2.68013 \\ \mathrm{C} & 1.05610 \\ \mathrm{C} & -1.29722 \\ \mathrm{C} & 1.09104 \\ \mathrm{C} & -1.28506 \\ \mathrm{C} & -0.14915 \\ \mathrm{C} & -0.11368 \\ \mathrm{H} & -3.10099 \\ \mathrm{H} & -3.05403 \\ \mathrm{H} & 2.08626 \\ \mathrm{H} & 3.18095 \\ \mathrm{H} & -3.24594 \\ \mathrm{H} & -2.08502 \\ \mathrm{H} & 3.75306 \\ \mathrm{H} & 2.38502 \\ \mathrm{H} & 2.43502 \\ \mathrm{H} & -0.20937 \\ \mathrm{H} & 1.90112 \\ \mathrm{H} & -2.22072 \\ \mathrm{H} & -0.14796 \\ \mathrm{H} & 1.96228 \\ \mathrm{H} & -2.20092 \\ \mathrm{H} & -2.14215 \\ \mathrm{H} & -3.26773 \\ \mathrm{H} & 3.17765 \\ \mathrm{H} & 2.07437\end{array}$

$$
\begin{array}{r}
-2.35369 \\
1.35564 \\
-2.38188 \\
-1.35182 \\
2.38908 \\
1.31683 \\
2.37279 \\
-0.07213 \\
-3.71198 \\
-3.73074 \\
3.77231 \\
3.74781 \\
-4.37400 \\
4.42205 \\
-0.84783 \\
0.90560 \\
1.21701 \\
1.70781 \\
-1.67986 \\
-1.22033 \\
-0.09798 \\
-0.97235 \\
0.79187 \\
-5.43415 \\
-4.24700 \\
-4.28012 \\
5.50255 \\
4.33974 \\
4.29504 \\
1.15034 \\
1.64484 \\
-1.66406 \\
-1.08315
\end{array}
$$

$$
\begin{array}{r}
-0.62023 \\
-0.87503 \\
0.12768 \\
0.63678 \\
-0.45417 \\
0.67347 \\
0.23688 \\
1.08383 \\
-0.88850 \\
-0.15318 \\
-0.54346 \\
0.11251 \\
-0.63466 \\
-0.25667 \\
-1.62788 \\
-1.62102 \\
-1.95431 \\
-0.64551 \\
0.44743 \\
1.71155 \\
0.87784 \\
1.61989 \\
1.69750 \\
-0.85354 \\
-1.30300 \\
-0.01881 \\
-0.34140 \\
-0.84520 \\
0.29781 \\
1.74689 \\
0.46673 \\
-0.77870 \\
-2.04204
\end{array}
$$

$\begin{array}{lrrr}\text { Mn-O: } & & & \\ \text { Mn } & 0.00000 & 0.00000 & 0.00000 \\ \mathrm{O} & 0.00000 & 0.00000 & 1.65461 \\ \mathrm{O} & -1.47960 & -0.06343 & -3.78044 \\ \mathrm{O} & -0.08094 & -0.01031 & -2.03686 \\ \mathrm{~N} & -0.04876 & -1.83314 & -0.21854 \\ \mathrm{~N} & 1.94694 & -0.00000 & -0.33737 \\ \mathrm{~N} & -1.98588 & 0.01006 & -0.18646 \\ \mathrm{~N} & -0.05828 & 1.83606 & -0.20358 \\ \mathrm{C} & -2.41034 & 0.02222 & -1.60832 \\ \mathrm{C} & -1.24008 & -0.02468 & -2.59855 \\ \mathrm{C} & 2.12069 & -1.29231 & -1.11530 \\ \mathrm{C} & 1.01462 & -2.32680 & -0.82838 \\ \mathrm{C} & 2.12500 & 1.31940 & -1.07194 \\ \mathrm{C} & -1.26640 & -2.34991 & -0.08995 \\ \mathrm{C} & -2.27418 & -1.32266 & 0.46016 \\ \mathrm{C} & 1.02642 & 2.35479 & -0.74727 \\ \mathrm{C} & -2.27904 & 1.32838 & 0.48259 \\ \mathrm{C} & -1.26346 & 2.36583 & -0.03333 \\ \mathrm{C} & 2.77374 & -0.04160 & 0.87913 \\ \mathrm{C} & 0.91481 & -3.64024 & -1.26230 \\ \mathrm{C} & -1.42761 & -3.64967 & -0.53138 \\ \mathrm{C} & 0.95695 & 3.70295 & -1.07033 \\ \mathrm{C} & -1.39729 & 3.69879 & -0.37655 \\ \mathrm{C} & -0.31014 & -4.28233 & -1.08559 \\ \mathrm{C} & -0.26062 & 4.35216 & -0.86293 \\ \mathrm{H} & -3.06874 & -0.82361 & -1.80760 \\ \mathrm{H} & -2.98768 & 0.92439 & -1.81360 \\ \mathrm{H} & 2.04753 & 1.12193 & -2.14141\end{array}$




$$
\begin{array}{r}
3.11719 \\
-3.30222 \\
-2.10574 \\
3.83304 \\
2.50335 \\
2.56772 \\
-0.41535 \\
1.73383 \\
-2.38163 \\
-0.33889 \\
1.79726 \\
-2.33939 \\
-2.12116 \\
-3.30553 \\
3.10857 \\
2.05452
\end{array}
$$

1.72179

$-1.63824$

$-1.21416$

$-0.08053$

$-0.91835$

0.83630

$-5.30107$

$-4.14344$

$-4.15885$

5.40042

4.23629

4.22397

1.20022

1.64832

$-1.70701$

$-1.06325$

\section{$F e-F$ :}

Fe

O

O

$\mathrm{N}$

$\mathrm{N}$

$\mathrm{N}$

$$
0.00000
$$$$
0.00000
$$$$
-1.44189
$$$$
-0.10576
$$$$
0.00017
$$$$
1.92775
$$$$
-1.95017
$$$$
-0.01780
$$$$
-2.44959
$$$$
-1.30233
$$$$
\text { 2. } 17175
$$$$
1.11832
$$$$
2.18052
$$$$
-1.17764
$$$$
-2.21407
$$$$
1.12051
$$$$
-2.23680
$$$$
-1.19158
$$$$
\text { 2. } 63377
$$$$
1.10311
$$$$
-1.25216
$$$$
1.12176
$$$$
-1.25172
$$$$
-0.09085
$$$$
-0.07517
$$$$
-3.08815
$$$$
-3.05275
$$$$
\text { 2.09229 }
$$$$
\text { 3.19072 }
$$$$
-3.23518
$$$$
-2.08748
$$$$
\text { 3. } 70993
$$$$
\text { 2. } 32862
$$$$
\text { 2. } 38170
$$$$
-0.12687
$$$$
1.96659
$$$$
-2.16272
$$$$
-0.09697
$$$$
\text { 2.00209 }
$$$$
-2.16070
$$$$
-2.14481
$$$$
-3.25379
$$$$
3.18551
$$$$
2.06795
$$

$$
\begin{aligned}
& 0.00000 \\
& 0.00000 \\
& -0.03459 \\
& 0.01467 \\
& -1.81171 \\
& -0.00092 \\
& -0.00757 \\
& \text { 1. } 81038 \\
& 0.00375 \\
& -0.00928 \\
& -1.29694 \\
& -2.34787 \\
& \text { 1. } 34702 \\
& -2.37264 \\
& -1.34390 \\
& \text { 2. } 37385 \\
& 1.30641 \\
& 2.35686 \\
& -0.07114 \\
& -3.72185 \\
& -3.73995 \\
& 3.75903 \\
& \text { 3. } 73669 \\
& -4.39117 \\
& 4.41271 \\
& -0.86049 \\
& 0.89409 \\
& \text { 1. } 21931 \\
& 1.68894 \\
& -1.67500 \\
& -1.20183 \\
& -0.09982 \\
& -0.96949 \\
& 0.79632 \\
& -5.46520 \\
& -4.26540 \\
& -4.29813 \\
& 5.49598 \\
& 4.32539 \\
& 4.28504 \\
& 1.13467 \\
& 1.63776 \\
& -1.65134 \\
& -1.09920 \\
& 0.00000 \\
& 1.74103 \\
& -3.57927 \\
& -1.80457 \\
& -0.07211 \\
& -0.17459 \\
& -0.01095 \\
& -0.01830 \\
& -1.40697 \\
& -2.40259 \\
& -0.93150 \\
& -0.54418 \\
& -0.83617 \\
& 0.18686 \\
& 0.66868 \\
& -0.39924 \\
& 0.69762 \\
& 0.28117 \\
& 1.12616 \\
& -0.71713 \\
& 0.00006 \\
& -0.43805 \\
& 0.21357 \\
& -0.43329 \\
& -0.13152 \\
& -1.59251 \\
& -1.58836 \\
& -1.91709 \\
& -0.60508 \\
& 0.47545 \\
& 1.74353 \\
& 0.93822 \\
& 1.65879 \\
& 1.73150 \\
& -0.57769 \\
& -1.07909 \\
& 0.17835 \\
& -0.17395 \\
& -0.71427 \\
& 0.42691 \\
& 1.77144 \\
& 0.48332 \\
& -0.73718 \\
& -1.99967
\end{aligned}
$$

$$
\begin{array}{r}
-0.85618 \\
0.27077 \\
1.53338 \\
0.60512 \\
1.46463 \\
1.48746 \\
-1.43859 \\
-1.75983 \\
-0.48308 \\
-1.12603 \\
-1.49576 \\
-0.28482 \\
1.55524 \\
0.29057 \\
-0.90303 \\
-2.17873
\end{array}
$$




\section{$\mathrm{Fe}-\mathrm{O}$ :}

\begin{tabular}{|c|c|c|c|}
\hline $\mathrm{Fe}$ & 0.00000 & 0.00000 & 0.00000 \\
\hline $\mathrm{O}$ & 0.00000 & 0.00000 & 1.62889 \\
\hline $\mathrm{O}$ & -1.41677 & -0.05974 & -3.74731 \\
\hline 0 & -0.04909 & -0.01195 & -1.98275 \\
\hline $\mathrm{N}$ & -0.04729 & -1.80594 & -0.18870 \\
\hline $\mathrm{N}$ & 1.92762 & -0.01435 & -0.28523 \\
\hline $\mathrm{N}$ & -1.95417 & 0.01707 & -0.16320 \\
\hline $\mathrm{N}$ & -0.03063 & 1.80898 & -0.18025 \\
\hline $\mathrm{C}$ & -2.37885 & 0.02443 & -1.58496 \\
\hline $\mathrm{C}$ & -1.20320 & -0.02259 & -2.56176 \\
\hline $\mathrm{C}$ & 2.12947 & -1.30364 & -1.05146 \\
\hline $\mathrm{C}$ & 1.02991 & -2.33207 & -0.74400 \\
\hline $\mathrm{C}$ & 2.15593 & 1.29959 & -1.00539 \\
\hline $\mathrm{C}$ & -1.25256 & -2.33822 & -0.02589 \\
\hline $\mathrm{C}$ & -2.26765 & -1.30376 & 0.48759 \\
\hline $\mathrm{C}$ & 1.07106 & 2.33985 & -0.67555 \\
\hline $\mathrm{C}$ & -2.25700 & 1.33534 & 0.49416 \\
\hline $\mathrm{C}$ & -1.22220 & 2.36264 & 0.00514 \\
\hline $\mathrm{C}$ & 2.71453 & -0.06565 & 0.95678 \\
\hline $\mathrm{C}$ & 0.94898 & -3.66980 & -1.10097 \\
\hline $\mathrm{C}$ & -1.39578 & -3.66612 & -0.38332 \\
\hline $\mathrm{C}$ & 1.03096 & 3.70147 & -0.94168 \\
\hline $\mathrm{C}$ & -1.32897 & 3.71183 & -0.27999 \\
\hline $\mathrm{C}$ & -0.26952 & -4.31605 & -0.89672 \\
\hline $\mathrm{C}$ & -0.17696 & 4.36490 & -0.72744 \\
\hline $\mathrm{H}$ & -3.03266 & -0.82471 & -1.78522 \\
\hline $\mathrm{H}$ & -2.95755 & 0.92435 & -1.79607 \\
\hline $\mathrm{H}$ & 2.08756 & 1.11356 & -2.07773 \\
\hline $\mathrm{H}$ & 3.15358 & 1.68163 & -0.77697 \\
\hline $\mathrm{H}$ & -3.29369 & -1.61677 & 0.28217 \\
\hline $\mathrm{H}$ & -2.12811 & -1.18688 & 1.56394 \\
\hline $\mathrm{H}$ & 3.78150 & -0.11155 & 0.71654 \\
\hline $\mathrm{H}$ & 2.42257 & -0.94403 & 1.52911 \\
\hline $\mathrm{H}$ & 2.49937 & 0.81292 & 1.56083 \\
\hline $\mathrm{H}$ & -0.35956 & -5.35580 & -1.18739 \\
\hline $\mathrm{H}$ & 1.78121 & -4.19251 & -1.55435 \\
\hline $\mathrm{H}$ & -2.34122 & -4.18604 & -0.29655 \\
\hline $\mathrm{H}$ & -0.23315 & 5.42476 & -0.94638 \\
\hline $\mathrm{H}$ & 1.88960 & 4.23573 & -1.32745 \\
\hline $\mathrm{H}$ & -2.26155 & 4.25015 & -0.16913 \\
\hline $\mathrm{H}$ & -2.13269 & 1.20940 & 1.57134 \\
\hline $\mathrm{H}$ & -3.27690 & 1.66306 & 0.28006 \\
\hline $\mathrm{H}$ & 3.12175 & -1.70833 & -0.83911 \\
\hline $\mathrm{H}$ & 2.05888 & -1.08375 & -2.1165 \\
\hline
\end{tabular}

$\begin{array}{lrrr}\text { Co-F: } & & & \\ \text { Co } & 0.00000 & 0.00000 & 0.00000 \\ \text { F } & 0.00000 & 0.00000 & 1.77282 \\ \text { O } & -1.42457 & -0.04775 & -3.53267 \\ \text { O } & -0.05352 & 0.01683 & -1.79608 \\ \mathrm{~N} & 0.01336 & -1.79109 & -0.02200 \\ \mathrm{~N} & 1.92012 & 0.00078 & -0.07989 \\ \mathrm{~N} & -1.91245 & -0.01228 & 0.04686 \\ \mathrm{~N} & -0.00449 & 1.78979 & 0.03486 \\ \mathrm{C} & -2.40801 & -0.00402 & -1.35238 \\ \mathrm{C} & -1.26824 & -0.01586 & -2.35618 \\ \mathrm{C} & 2.19827 & -1.29244 & -0.81837 \\ \mathrm{C} & 1.16425 & -2.33953 & -0.39697 \\ \mathrm{C} & 2.20041 & 1.33769 & -0.73832 \\ \mathrm{C} & -1.13950 & -2.36219 & 0.30968 \\ \mathrm{C} & -2.18723 & -1.33238 & 0.74240 \\ \mathrm{C} & 1.15208 & 2.35870 & -0.28592\end{array}$




$\begin{array}{lr}\mathrm{C} & -2.21289 \\ \mathrm{C} & -1.16283 \\ \mathrm{C} & 2.58308 \\ \mathrm{C} & 1.19658 \\ \mathrm{C} & -1.16451 \\ \mathrm{C} & 1.17774 \\ \mathrm{C} & -1.19481 \\ \mathrm{C} & 0.01933 \\ \mathrm{C} & -0.00849 \\ \mathrm{H} & -3.04550 \\ \mathrm{H} & -3.01360 \\ \mathrm{H} & 2.11116 \\ \mathrm{H} & 3.21383 \\ \mathrm{H} & -3.20482 \\ \mathrm{H} & -2.07588 \\ \mathrm{H} & 3.66425 \\ \mathrm{H} & 2.26171 \\ \mathrm{H} & 2.31568 \\ \mathrm{H} & 0.02248 \\ \mathrm{H} & 2.08963 \\ \mathrm{H} & -2.05602 \\ \mathrm{H} & -0.00923 \\ \mathrm{H} & 2.07325 \\ \mathrm{H} & -2.09246 \\ \mathrm{H} & -2.13483 \\ \mathrm{H} & -3.22693 \\ \mathrm{H} & 3.21776 \\ \mathrm{H} & 2.08816\end{array}$

$\begin{array}{rr}1.28866 & 0.76410 \\ 2.33796 & 0.38471 \\ -0.05852 & 1.24150 \\ -3.72254 & -0.41463 \\ -3.74430 & 0.27027 \\ 3.74135 & -0.23279 \\ 3.72012 & 0.41235 \\ -4.39981 & -0.08303 \\ 4.39670 & 0.10972 \\ -0.86936 & -1.53685 \\ 0.88459 & -1.53495 \\ 1.21820 & -1.82034 \\ 1.66670 & -0.50252 \\ -1.66865 & 0.53843 \\ -1.17132 & 1.81597 \\ -0.08984 & 1.08283 \\ -0.94977 & 1.77467 \\ 0.81322 & 1.83269 \\ -5.48389 & -0.10600 \\ -4.26941 & -0.68971 \\ -4.30842 & 0.51327 \\ 5.48055 & 0.14298 \\ 4.30532 & -0.46132 \\ 4.26731 & 0.67141 \\ 1.10474 & 1.83657 \\ 1.62144 & 0.53724 \\ -1.62598 & -0.61780 \\ -1.11620 & -1.88986\end{array}$

$\begin{array}{lrrr}\mathrm{CO}-\mathrm{O}: & & & \\ \mathrm{CO} & 0.00000 & 0.00000 & 0.00000 \\ \mathrm{O} & 0.00000 & 0.00000 & 1.78973 \\ \mathrm{O} & -1.46657 & -0.06435 & -3.63965 \\ \mathrm{O} & -0.04559 & 0.00206 & -1.92146 \\ \mathrm{~N} & -0.00759 & -1.78560 & -0.07954 \\ \mathrm{~N} & 1.91924 & -0.00158 & -0.14858 \\ \mathrm{~N} & -1.91650 & -0.00262 & -0.04184 \\ \mathrm{~N} & -0.02121 & 1.78671 & -0.03711 \\ \mathrm{C} & -2.37385 & 0.00481 & -1.45404 \\ \mathrm{C} & -1.22209 & -0.02531 & -2.46086 \\ \mathrm{C} & 2.16017 & -1.27638 & -0.91791 \\ \mathrm{C} & 1.09582 & -2.32312 & -0.57054 \\ \mathrm{C} & 2.16854 & 1.31916 & -0.83719 \\ \mathrm{C} & -1.18775 & -2.34782 & 0.14302 \\ \mathrm{C} & -2.21222 & -1.31595 & 0.62948 \\ \mathrm{C} & 1.09971 & 2.35008 & -0.45037 \\ \mathrm{C} & -2.23131 & 1.29535 & 0.64700 \\ \mathrm{C} & -1.19927 & 2.34256 & 0.20935 \\ \mathrm{C} & 2.65654 & -0.06543 & 1.12173 \\ \mathrm{C} & 1.06467 & -3.68797 & -0.80915 \\ \mathrm{C} & -1.28279 & -3.70565 & -0.10356 \\ \mathrm{C} & 1.08500 & 3.73121 & -0.57682 \\ \mathrm{C} & -1.28163 & 3.71536 & 0.05477 \\ \mathrm{C} & -0.13415 & -4.35642 & -0.56063 \\ \mathrm{C} & -0.11716 & 4.39011 & -0.31990 \\ \mathrm{H} & -3.02364 & -0.85012 & -1.64275 \\ \mathrm{H} & -2.96511 & 0.90031 & -1.64834 \\ \mathrm{H} & 2.07523 & 1.16202 & -1.91315 \\ \mathrm{H} & 3.17517 & 1.68176 & -0.61652 \\ \mathrm{H} & -3.23761 & -1.63988 & 0.43998 \\ \mathrm{H} & -2.06341 & -1.17384 & 1.70164 \\ \mathrm{H} & 3.73071 & -0.10590 & 0.91412 \\ \mathrm{H} & 2.34763 & -0.94916 & 1.67548\end{array}$




$\begin{array}{rrrr}\mathrm{H} & 2.41794 & 0.80240 & 1.73104 \\ \mathrm{H} & -0.18533 & -5.42067 & -0.75757 \\ \mathrm{H} & 1.92301 & -4.21949 & -1.19892 \\ \mathrm{H} & -2.20621 & -4.25135 & 0.04086 \\ \mathrm{H} & -0.15437 & 5.46716 & -0.43446 \\ \mathrm{H} & 1.96037 & 4.28569 & -0.88915 \\ \mathrm{H} & -2.20505 & 4.25549 & 0.21920 \\ \mathrm{H} & -2.11120 & 1.13557 & 1.71992 \\ \mathrm{H} & -3.25341 & 1.61823 & 0.43809 \\ \mathrm{H} & 3.16908 & -1.64937 & -0.72676 \\ \mathrm{H} & 2.05509 & -1.06058 & -1.98172\end{array}$

\section{$\mathrm{Ni}-\mathrm{F}$ :}

$\mathrm{Ni}$

$\mathrm{F}$

O

$\mathrm{O}$

N

$\mathrm{N}$

$\mathrm{N}$

C

C

C

C

C

C

C

C

C

C

C

C

C

C

C

C

C

$\mathrm{H}$

$\mathrm{H}$

$\mathrm{H}$

$\mathrm{H}$

$\mathrm{H}$

$\mathrm{H}$

$\mathrm{H}$

$\mathrm{H}$

$\mathrm{H}$
0.00000

0.00000

$-0.04730$

0.01086

$-1.77917$

0.00062

$-0.01252$

1.77744

$-0.00319$

$-0.01786$

$-1.28712$

$-2.32961$

1.33542

$-2.35436$

$-1.33010$

2.35079

1.28309

2.32844

$-0.05970$

$-3.71308$

$-3.73619$

3. 73417

3. 71113

$-4.39288$

4.39082

$-0.86864$

0.88879

1.20869

1.65866

$-1.65518$

$-1.16689$

$-0.08730$

$-0.95363$

0.81036

$-5.47640$

$-4.25550$

$-4.29681$

5.47461

4.29555

4.25404

1.09480

1.60584

$-1.61590$

$-1.09983$
0.00000

1.78944

$-3.53811$

$-1.82636$

$-0.03568$

$-0.09742$

0.04875

0.02292

$-1.34731$

$-2.36339$

$-0.84517$

$-0.43184$

$-0.76116$

0.30032

0.74928

$-0.30909$

0.77411

0.38463

1.22756

$-0.46720$

0.24234

$-0.25821$

0.40842

$-0.13226$

0.09342

$-1.52425$

$-1.52267$

$-1.84190$

$-0.52166$

0.55170

1.82051

1.06721

1.75622

1.81844

$-0.17029$

$-0.75924$

0.48687

0.12355

$-0.49535$

0.67517

1.84444

0.55039

$-0.63692$

$-1.91473$

$$
\begin{array}{r}
0.00000 \\
1.79246 \\
-3.68643 \\
-1.90729
\end{array}
$$




$\begin{array}{lr}\mathrm{N} & -0.01094 \\ \mathrm{~N} & 2.04533 \\ \mathrm{~N} & -2.06404 \\ \mathrm{~N} & -0.02518 \\ \mathrm{C} & -2.42354 \\ \mathrm{C} & -1.21349 \\ \mathrm{C} & 2.19053 \\ \mathrm{C} & 1.08559 \\ \mathrm{C} & 2.20206 \\ \mathrm{C} & -1.21310 \\ \mathrm{C} & -2.27832 \\ \mathrm{C} & 1.09227 \\ \mathrm{C} & -2.29869 \\ \mathrm{C} & -1.22336 \\ \mathrm{C} & 2.78775 \\ \mathrm{C} & 1.02853 \\ \mathrm{C} & -1.32542 \\ \mathrm{C} & 1.05637 \\ \mathrm{C} & -1.31766 \\ \mathrm{C} & -0.18137 \\ \mathrm{C} & -0.15484 \\ \mathrm{H} & -3.05052 \\ \mathrm{H} & -3.01246 \\ \mathrm{H} & 2.11599 \\ \mathrm{H} & 3.18603 \\ \mathrm{H} & -3.27947 \\ \mathrm{H} & -2.15334 \\ \mathrm{H} & 3.86346 \\ \mathrm{H} & 2.45511 \\ \mathrm{H} & 2.58156 \\ \mathrm{H} & -0.24992 \\ \mathrm{H} & 1.87843 \\ \mathrm{H} & -2.26549 \\ \mathrm{H} & -0.20449 \\ \mathrm{H} & 1.92603 \\ \mathrm{H} & -2.25458 \\ \mathrm{H} & -2.20857 \\ \mathrm{H} & -3.29569 \\ \mathrm{H} & 3.17631 \\ \mathrm{H} & 2.09084 \\ & \end{array}$

$$
\begin{array}{r}
-1.78319 \\
-0.00266 \\
0.00038 \\
1.78354 \\
0.00848 \\
-0.00932 \\
-1.27379 \\
-2.29881 \\
1.31885 \\
-2.32567 \\
-1.31383 \\
2.32959 \\
1.29924 \\
2.32540 \\
-0.07826 \\
-3.64567 \\
-3.66367 \\
3.70249 \\
3.68705 \\
-4.30846 \\
4.35912 \\
-0.85126 \\
0.89616 \\
1.17392 \\
1.74630 \\
-1.70836 \\
-1.17431 \\
-0.15502 \\
-0.94708 \\
0.80071 \\
-5.35605 \\
-4.16146 \\
-4.19396 \\
5.42801 \\
4.24909 \\
4.21812 \\
1.14123 \\
1.69450 \\
-1.71410 \\
-1.06246
\end{array}
$$

$$
\begin{array}{r}
-0.09532 \\
-0.19565 \\
-0.13553 \\
-0.04747 \\
-1.55545 \\
-2.49397 \\
-0.95797 \\
-0.62565 \\
-0.86740 \\
0.05309 \\
0.52470 \\
-0.49289 \\
0.54422 \\
0.12874 \\
1.06135 \\
-0.95165 \\
-0.27729 \\
-0.69361 \\
-0.09721 \\
-0.75430 \\
-0.48152 \\
-1.79903 \\
-1.79473 \\
-1.94597 \\
-0.65574 \\
0.33081 \\
1.60044 \\
0.86481 \\
1.62765 \\
1.66877 \\
-1.02254 \\
-1.37981 \\
-0.19420 \\
-0.65404 \\
-1.03510 \\
0.01287 \\
1.62066 \\
0.33017 \\
-0.78324 \\
-2.02360
\end{array}
$$

$\begin{array}{lrrr}\text { TC-F: } & & & \\ \text { TC } & 0.00000 & 0.00000 & 0.00000 \\ \text { F } & 0.00000 & 0.00000 & 1.87929 \\ \text { O } & -1.39264 & 0.48263 & -3.74834 \\ \text { O } & -0.11069 & 0.00447 & -1.98579 \\ \text { N } & -0.07243 & -1.92812 & -0.21133 \\ \mathrm{~N} & 2.00756 & -0.00585 & -0.39769 \\ \mathrm{~N} & -2.06718 & -0.01202 & -0.24110 \\ \mathrm{~N} & -0.06388 & 1.93150 & -0.22444 \\ \mathrm{C} & -2.46328 & -0.00718 & -1.67193 \\ \mathrm{C} & -1.27032 & 0.20377 & -2.59813 \\ \mathrm{C} & 2.12490 & -1.35178 & -1.15256 \\ \mathrm{C} & 0.99372 & -2.39167 & -0.86101 \\ \mathrm{C} & 2.13558 & 1.33518 & -1.16152 \\ \mathrm{C} & -1.31848 & -2.41433 & -0.13540 \\ \mathrm{C} & -2.34073 & -1.37583 & 0.41190 \\ \mathrm{C} & 0.98612 & 2.36790 & -0.91960 \\ \mathrm{C} & -2.33296 & 1.36515 & 0.39034 \\ \mathrm{C} & -1.32424 & 2.39335 & -0.19774 \\ \mathrm{C} & 2.89379 & -0.00728 & 0.79081 \\ \mathrm{C} & 0.87246 & -3.68544 & -1.34477 \\ \mathrm{C} & -1.49068 & -3.68983 & -0.63098\end{array}$




$\begin{array}{lr}\mathrm{C} & 0.83582 \\ \mathrm{C} & -1.52536 \\ \mathrm{C} & -0.36517 \\ \mathrm{C} & -0.41364 \\ \mathrm{H} & -2.91235 \\ \mathrm{H} & -3.21818 \\ \mathrm{H} & 2.12497 \\ \mathrm{H} & 3.10133 \\ \mathrm{H} & -3.36376 \\ \mathrm{H} & -2.21166 \\ \mathrm{H} & 3.93720 \\ \mathrm{H} & 2.69062 \\ \mathrm{H} & 2.69708 \\ \mathrm{H} & -0.47858 \\ \mathrm{H} & 1.67999 \\ \mathrm{H} & -2.45052 \\ \mathrm{H} & -0.55061 \\ \mathrm{H} & 1.62952 \\ \mathrm{H} & -2.49970 \\ \mathrm{H} & -2.18461 \\ \mathrm{H} & -3.36025 \\ \mathrm{H} & 3.10305 \\ \mathrm{H} & 2.07374\end{array}$

$$
\begin{array}{r}
3.61859 \\
3.62169 \\
-4.31708 \\
4.23420 \\
-0.96640 \\
0.75658 \\
1.11714 \\
1.77229 \\
-1.69524 \\
-1.26860 \\
-0.01192 \\
-0.89096 \\
0.87918 \\
-5.32423 \\
-4.18802 \\
-4.19149 \\
5.20472 \\
4.09385 \\
4.09273 \\
1.27834 \\
1.67687 \\
-1.77888 \\
-1.14029
\end{array}
$$

$$
\begin{array}{r}
-1.49933 \\
-0.78993 \\
-1.18800 \\
-1.38897 \\
-1.93509 \\
-1.85757 \\
-2.22927 \\
-0.90367 \\
0.20785 \\
1.49164 \\
0.46543 \\
1.39470 \\
1.39246 \\
-1.57236 \\
-1.86266 \\
-0.62335 \\
-1.85143 \\
-2.06236 \\
-0.83077 \\
1.46925 \\
0.19718 \\
-0.92667 \\
-2.22074
\end{array}
$$

$\begin{array}{lrrr}\text { TC-O: } & & & \\ \text { TC } & 0.00000 & 0.00000 & 0.00000 \\ \mathrm{O} & 0.00000 & 0.00000 & 1.68692 \\ \mathrm{O} & -1.36163 & 0.52149 & -4.00905 \\ \mathrm{O} & -0.09197 & 0.01459 & -2.23728 \\ \mathrm{~N} & -0.10065 & -1.89871 & -0.48054 \\ \mathrm{~N} & 1.93024 & -0.00194 & -0.72657 \\ \mathrm{~N} & -2.04503 & -0.00777 & -0.50813 \\ \mathrm{~N} & -0.10164 & 1.90763 & -0.47870 \\ \mathrm{C} & -2.41009 & 0.00736 & -1.94329 \\ \mathrm{C} & -1.19983 & 0.22422 & -2.85011 \\ \mathrm{C} & 2.04382 & -1.30725 & -1.50991 \\ \mathrm{C} & 0.89211 & -2.30847 & -1.27804 \\ \mathrm{C} & 2.06148 & 1.32051 & -1.48144 \\ \mathrm{C} & -1.36783 & -2.36043 & -0.51938 \\ \mathrm{C} & -2.37475 & -1.36778 & 0.09178 \\ \mathrm{C} & 0.88111 & 2.29872 & -1.29780 \\ \mathrm{C} & -2.37103 & 1.35529 & 0.08953 \\ \mathrm{C} & -1.37811 & 2.34438 & -0.54756 \\ \mathrm{C} & 2.90149 & -0.02651 & 0.39110 \\ \mathrm{C} & 0.70158 & -3.48318 & -1.98651 \\ \mathrm{C} & -1.61563 & -3.50684 & -1.24127 \\ \mathrm{C} & 0.66818 & 3.43084 & -2.06720 \\ \mathrm{C} & -1.64805 & 3.44544 & -1.32864 \\ \mathrm{C} & -0.54500 & -4.09940 & -1.91977 \\ \mathrm{C} & -0.58879 & 4.02517 & -2.03438 \\ \mathrm{H} & -2.84925 & -0.95159 & -2.21925 \\ \mathrm{H} & -3.16419 & 0.77109 & -2.12919 \\ \mathrm{H} & 2.10822 & 1.10264 & -2.54685 \\ \mathrm{H} & 3.00343 & 1.78745 & -1.18548 \\ \mathrm{H} & -3.40031 & -1.66256 & -0.14282 \\ \mathrm{H} & -2.25532 & -1.30970 & 1.17509 \\ \mathrm{H} & 3.91859 & -0.02710 & -0.01264 \\ \mathrm{H} & 2.74343 & -0.92248 & 0.98891 \\ \mathrm{H} & 2.75562 & 0.84825 & 1.02196 \\ \mathrm{H} & -0.71376 & -5.01148 & -2.47808 \\ \mathrm{H} & 1.47738 & -3.88825 & -2.62436 \\ \mathrm{H} & -2.61220 & -3.92346 & -1.31744 \\ \mathrm{H} & -0.77616 & 4.89968 & -2.64434\end{array}$




$\begin{array}{rrrr}\mathrm{H} & 1.43530 & 3.81357 & -2.72857 \\ \mathrm{H} & -2.65425 & 3.83081 & -1.43494 \\ \mathrm{H} & -2.23173 & 1.30416 & 1.17054 \\ \mathrm{H} & -3.40172 & 1.64308 & -0.12959 \\ \mathrm{H} & 3.01105 & -1.76303 & -1.28659 \\ \mathrm{H} & 2.01430 & -1.06376 & -2.57032\end{array}$

$\mathrm{H} \quad 2.01430$

$-1.06376$

$-2.57032$

\section{Ru-F:}

\begin{tabular}{|c|c|}
\hline $\mathrm{Ru}$ & 0.00000 \\
\hline $\mathrm{F}$ & 0.00000 \\
\hline 0 & -1.35567 \\
\hline 0 & -0.09100 \\
\hline $\mathrm{N}$ & -0.04232 \\
\hline $\mathrm{N}$ & 1.98457 \\
\hline $\mathrm{N}$ & -2.03874 \\
\hline $\mathrm{N}$ & -0.04205 \\
\hline $\mathrm{C}$ & -2.44971 \\
\hline $\mathrm{C}$ & -1.25178 \\
\hline $\mathrm{C}$ & 2.15874 \\
\hline $\mathrm{C}$ & 1.03900 \\
\hline $\mathrm{C}$ & 2.15884 \\
\hline $\mathrm{C}$ & -1.28055 \\
\hline $\mathrm{C}$ & -2.31199 \\
\hline $\mathrm{C}$ & 1.04076 \\
\hline $\mathrm{C}$ & -2.31134 \\
\hline $\mathrm{C}$ & -1.27893 \\
\hline $\mathrm{C}$ & 2.80141 \\
\hline $\mathrm{C}$ & 0.93529 \\
\hline $\mathrm{C}$ & -1.43476 \\
\hline $\mathrm{C}$ & 0.93991 \\
\hline $\mathrm{C}$ & -1.43038 \\
\hline $\mathrm{C}$ & -0.29833 \\
\hline $\mathrm{C}$ & -0.29265 \\
\hline $\mathrm{H}$ & -3.06142 \\
\hline $\mathrm{H}$ & -3.05830 \\
\hline $\mathrm{H}$ & 2.11553 \\
\hline $\mathrm{H}$ & 3.14388 \\
\hline $\mathrm{H}$ & -3.33280 \\
\hline $\mathrm{H}$ & -2.19028 \\
\hline $\mathrm{H}$ & 3.86015 \\
\hline $\mathrm{H}$ & 2.57139 \\
\hline $\mathrm{H}$ & 2.57161 \\
\hline $\mathrm{H}$ & -0.39829 \\
\hline $\mathrm{H}$ & 1.75388 \\
\hline $\mathrm{H}$ & -2.38686 \\
\hline $\mathrm{H}$ & -0.39035 \\
\hline $\mathrm{H}$ & 1.75997 \\
\hline $\mathrm{H}$ & -2.38110 \\
\hline $\mathrm{H}$ & -2.19011 \\
\hline $\mathrm{H}$ & -3.33187 \\
\hline $\mathrm{H}$ & 3.14286 \\
\hline $\mathrm{H}$ & 2.11795 \\
\hline
\end{tabular}

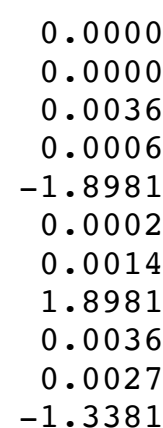

3816

$-2.38070$

1.34007

$-2.40129$

$-1.36691$

2. 38300

1. 36794

2.40325

$-0.00106$

$-3.69090$

$-3.69700$

3.69669

3. 70265

$-4.32853$

4.33547

$-0.87114$

0.88117

1.14138

1.74637

$-1.69021$

$-1.25940$

$-0.00099$

$-0.88748$

0.88427

$-5.34858$

$-4.20320$

$-4.21236$

5.35846

4.21134

4.22029

1.25742

1.69247

$-1.74599$

$-1.13725$
0.00000

1.84757

$-3.77175$

$-1.93832$

$-0.20420$

$-0.36705$

$-0.22532$

$-0.20169$

$-1.64939$

$-2.58879$

$-1.10752$

$-0.81320$

$-1.10499$

$-0.11593$

0.41716

$-0.80599$

0.42092

$-0.10861$

0.87117

$-1.25555$

$-0.56275$

$-1.23858$

$-0.54591$

$-1.09249$

$-1.07118$

$-1.87117$

$-1.86921$

$-2.17656$

$-0.86952$

0.20835

1.49727

0.59989

1.46080

1.46249

$-1.44547$

$-1.74557$

$-0.53832$

$-1.41626$

$-1.72359$

$-0.51652$

1. 50077

0.21261

$-0.87087$

$-2.17876$
0.00000
0.00000
-0.00424
-0.00052
-1.89520
-0.00045
0.00142
1.89520
0.00236
0.00000

1.75269

$-3.89731$

$-2.10861$

$-0.29134$

$-0.40798$

$-0.31622$

$-0.29066$

$-1.75845$ 


\section{Rh-F:}

$\begin{array}{lr}\mathrm{Rh} & 0.00000 \\ \mathrm{~F} & 0.00000 \\ \mathrm{O} & -1.58538 \\ \mathrm{O} & -0.11584 \\ \mathrm{~N} & 0.01498 \\ \mathrm{~N} & 2.00808 \\ \mathrm{~N} & -1.99208 \\ \mathrm{~N} & 0.01357 \\ \mathrm{C} & -2.48494 \\ \mathrm{C} & -1.36829 \\ \mathrm{C} & 2.25548 \\ \mathrm{C} & 1.20312 \\ \mathrm{C} & 2.25537 \\ \mathrm{C} & -1.15584 \\ \mathrm{C} & -2.23402 \\ \mathrm{C} & 1.20268 \\ \mathrm{C} & -2.23553 \\ \mathrm{C} & -1.15649 \\ \mathrm{C} & 2.64134 \\ \mathrm{C} & 1.24488 \\ \mathrm{C} & -1.16356 \\ \mathrm{C} & 1.24537 \\ \mathrm{C} & -1.16312 \\ \mathrm{C} & 0.04940 \\ \mathrm{C} & 0.05018 \\ \mathrm{H} & -3.10993\end{array}$

$$
\begin{array}{r}
0.00000 \\
1.90473 \\
-3.57206 \\
-1.90746 \\
0.04981 \\
-0.00270 \\
0.03786 \\
0.05312 \\
-1.36350 \\
-2.40413 \\
-0.68054 \\
-0.22864 \\
-0.67541 \\
0.38288 \\
0.74650 \\
-0.21975 \\
0.74899 \\
0.39113 \\
1.33217 \\
-0.14960 \\
0.43359 \\
-0.13093 \\
0.45224 \\
0.17369 \\
0.19664 \\
-1.54393
\end{array}
$$




$\begin{array}{lr}\mathrm{H} & -3.10975 \\ \mathrm{H} & 2.14339 \\ \mathrm{H} & 3.26966 \\ \mathrm{H} & -3.23991 \\ \mathrm{H} & -2.14627 \\ \mathrm{H} & 3.72680 \\ \mathrm{H} & 2.33285 \\ \mathrm{H} & 2.33359 \\ \mathrm{H} & 0.06449 \\ \mathrm{H} & 2.14778 \\ \mathrm{H} & -2.05067 \\ \mathrm{H} & 0.06609 \\ \mathrm{H} & 2.14885 \\ \mathrm{H} & -2.04962 \\ \mathrm{H} & -2.15127 \\ \mathrm{H} & -3.24075 \\ \mathrm{H} & 3.27000 \\ \mathrm{H} & 2.14282\end{array}$

$$
\begin{array}{r}
0.87557 \\
1.22435 \\
1.69764 \\
-1.70713 \\
-1.15822 \\
-0.00301 \\
-0.88507 \\
0.87768 \\
-5.53170 \\
-4.35613 \\
-4.36342 \\
5.52985 \\
4.35693 \\
4.35990 \\
1.15266 \\
1.70445 \\
-1.69532 \\
-1.21767
\end{array}
$$

$$
\begin{array}{r}
-1.54355 \\
-1.75485 \\
-0.46590 \\
0.53285 \\
1.81863 \\
1.20415 \\
1.88876 \\
1.89213 \\
0.22862 \\
-0.33961 \\
0.67898 \\
0.25961 \\
-0.31608 \\
0.70253 \\
1.82097 \\
0.53307 \\
-0.47291 \\
-1.75943
\end{array}
$$

$\begin{array}{lrrr}\text { Rh-O: } & & & \\ \mathrm{Rh} & 0.00000 & 0.00000 & 0.00000 \\ \mathrm{O} & 0.00000 & 0.00000 & 1.89164 \\ \mathrm{O} & -1.52346 & 0.00796 & -3.74963 \\ \mathrm{O} & -0.07160 & 0.00086 & -2.05700 \\ \mathrm{~N} & -0.03773 & -1.87801 & -0.13593 \\ \mathrm{~N} & 1.99696 & -0.00721 & -0.20122 \\ \mathrm{~N} & -2.00417 & 0.00764 & -0.13502 \\ \mathrm{~N} & -0.02396 & 1.87830 & -0.13371 \\ \mathrm{C} & -2.41623 & 0.00981 & -1.56415 \\ \mathrm{C} & -1.25941 & 0.00617 & -2.57423 \\ \mathrm{C} & 2.17197 & -1.32526 & -0.95800 \\ \mathrm{C} & 1.05975 & -2.37815 & -0.67939 \\ \mathrm{C} & 2.18221 & 1.31140 & -0.95473 \\ \mathrm{C} & -1.26119 & -2.38730 & -0.01293 \\ \mathrm{C} & -2.27950 & -1.33832 & 0.51325 \\ \mathrm{C} & 1.07806 & 2.37207 & -0.67363 \\ \mathrm{C} & -2.26978 & 1.35471 & 0.51472 \\ \mathrm{C} & -1.24327 & 2.39694 & -0.00899 \\ \mathrm{C} & 2.79003 & -0.01205 & 1.03522 \\ \mathrm{C} & 0.97724 & -3.71068 & -1.06121 \\ \mathrm{C} & -1.39681 & -3.70738 & -0.40391 \\ \mathrm{C} & 1.00667 & 3.70700 & -1.04933 \\ \mathrm{C} & -1.36801 & 3.71975 & -0.39447 \\ \mathrm{C} & -0.25380 & -4.34960 & -0.89611 \\ \mathrm{C} & -0.21943 & 4.35500 & -0.88264 \\ \mathrm{H} & -3.03673 & -0.86317 & -1.76830 \\ \mathrm{H} & -3.03169 & 0.88650 & -1.76759 \\ \mathrm{H} & 2.11571 & 1.09120 & -2.02104 \\ \mathrm{H} & 3.17362 & 1.71641 & -0.73970 \\ \mathrm{H} & -3.30634 & -1.66417 & 0.33403 \\ \mathrm{H} & -2.10990 & -1.21532 & 1.58550 \\ \mathrm{H} & 3.85674 & -0.01568 & 0.78891 \\ \mathrm{H} & 2.53551 & -0.89012 & 1.62545 \\ \mathrm{H} & 2.54187 & 0.86589 & 1.62830 \\ \mathrm{H} & -0.34005 & -5.38438 & -1.20521 \\ \mathrm{H} & 1.81280 & -4.23790 & -1.50351 \\ \mathrm{H} & -2.34298 & -4.23103 & -0.35734 \\ \mathrm{H} & -0.29699 & 5.39196 & -1.18676 \\ \mathrm{H} & 1.84710 & 4.22976 & -1.48764 \\ \mathrm{H} & -2.30986 & 4.25093 & -0.34577 \\ \mathrm{H} & -2.10204 & 1.22917 & 1.58697 \\ \mathrm{H} & -3.29401 & 1.68848 & 0.33515 \\ \mathrm{H} & 3.16031 & -1.73841 & -0.74436 \\ & & & \\ & & & \end{array}$




\section{Pd-F:}

$\begin{array}{lr}\mathrm{F} & 0.00000 \\ \mathrm{P} & 0.00000 \\ \mathrm{P} & -1.56636\end{array}$

0.00000
0.00000
-0.00809
-0.00090
-1.86940
-0.00000
0.00064
1.86933
0.00465
-0.00308

0.00000

$-1.56636$

$-0.09802$

0.00010

1.98742

$-1.97979$

$-0.00057$

$-2.45891$

$-1.33597$

2.22374

1.16639

2.22310

$-1.17531$

$-2.23048$

1.16634

$-2.22986$

$-1.17480$

2.63757

1.18758

$-1.20654$

1.18875

$-1.20476$

$-0.01015$

$-0.00823$

$-3.08990$

$-3.07200$

2. 11658

3.23601

$-3.24676$

$-2.11402$

3.72064

2.33706

2.33601

$-0.01300$

2.08048

$-2.10461$

$-0.01016$

2.08207

$-2.10198$

$-2.11214$

$-3.24636$

3.23640

2. 11846

$-1.34574$

$-2.40226$

1.34707

$-2.40458$

$-1.34427$

2.40280

1. 34260

2.40402

$-0.00094$

$-3.78861$

$-3.78938$

3. 78910

3. 78902

$-4.44899$

4.44906

$-0.86645$

0.88872

1.18859

1.69221

$-1.68389$

$-1.17229$

$-0.00017$

$-0.88392$

0.88054

$-5.53324$

$-4.35243$

$-4.35380$

5.53336

4.35327

4.35317

1.16683

1.68287

$-1.69129$

$-1.18539$

1.92298

$-3.60529$

$-1.95166$

$-0.01208$

$-0.06834$

0.00856

$-0.00924$

$-1.40021$

$-2.43966$

$-0.77080$

$-0.35020$

$-0.76856$

0.29233

0.70883

$-0.34450$

0.71441

0.30029

1.25723

$-0.35158$

0.25778

$-0.33925$

0.27250

$-0.05196$

$-0.03572$

$-1.57807$

$-1.57706$

$-1.84379$

$-0.55644$

0.50678

1.78046

1.10835

1.81718

1.81895

$-0.06498$

$-0.59030$

0.47453

$-0.04349$

$-0.57559$

0.49343

1.78524

0.51449

$-0.55819$

$-1.84585$

$\begin{array}{lr}P d-O: & \\ \text { Pd } & 0.00000 \\ O & 0.00000 \\ O & -1.79483 \\ O & -0.16286 \\ \mathrm{~N} & -0.00938 \\ \mathrm{~N} & 1.98553 \\ \mathrm{~N} & -1.97644 \\ \mathrm{~N} & -0.00720 \\ \mathrm{C} & -2.47259 \\ \mathrm{C} & -1.39331 \\ \mathrm{C} & 2.18300 \\ \mathrm{C} & 1.11998 \\ \mathrm{C} & 2.18459 \\ \mathrm{C} & -1.19996\end{array}$

0.00000
0.00000
-0.01145
-0.00020
-1.86420
-0.00112
0.00138
1.86418
0.00189
-0.00416
-1.32602
-2.39052
1.32452
-2.39062

0.00000

1.89896

$-3.64626$

$-2.12283$

$-0.03524$

$-0.12362$

0.00347

$-0.03356$

$-1.39789$

$-2.51018$

$-0.85952$

$-0.47249$

$-0.85761$

0.21921 


$\begin{array}{lr}\mathrm{C} & -2.20728 \\ \mathrm{C} & 1.12362 \\ \mathrm{C} & -2.20590 \\ \mathrm{C} & -1.19664 \\ \mathrm{C} & 2.68469 \\ \mathrm{C} & 1.09856 \\ \mathrm{C} & -1.27638 \\ \mathrm{C} & 1.10534 \\ \mathrm{C} & -1.27015 \\ \mathrm{C} & -0.10973 \\ \mathrm{C} & -0.10185 \\ \mathrm{H} & -3.10528 \\ \mathrm{H} & -3.09705 \\ \mathrm{H} & 2.04588 \\ \mathrm{H} & 3.19745 \\ \mathrm{H} & -3.24164 \\ \mathrm{H} & -1.94287 \\ \mathrm{H} & 3.76456 \\ \mathrm{H} & 2.37998 \\ \mathrm{H} & 2.37329 \\ \mathrm{H} & -0.15006 \\ \mathrm{H} & 1.96285 \\ \mathrm{H} & -2.19177 \\ \mathrm{H} & -0.13973 \\ \mathrm{H} & 1.97124 \\ \mathrm{H} & -2.18448 \\ \mathrm{H} & -1.94268 \\ \mathrm{H} & -3.23970 \\ \mathrm{H} & 3.19517 \\ \mathrm{H} & 2.04521\end{array}$

$$
\begin{array}{r}
-1.32867 \\
2.38990 \\
1.33087 \\
2.39208 \\
-0.00155 \\
-3.76915 \\
-3.76257 \\
3.76919 \\
3.76487 \\
-4.42210 \\
4.42362 \\
-0.87195 \\
0.88126 \\
1.13150 \\
1.69279 \\
-1.65618 \\
-1.14191 \\
0.00272 \\
-0.87574 \\
0.86775 \\
-5.49675 \\
-4.32771 \\
-4.31600 \\
5.49890 \\
4.32723 \\
4.31953 \\
1.14265 \\
1.65998 \\
-1.69623 \\
-1.13098
\end{array}
$$

0.71443

$-0.46777$

0.71579

0.22274

1.16840

$-0.62583$

0.04010

$-0.61522$

0.04858

$-0.36220$

$-0.35021$

$-1.55338$

$-1.55560$

$-1.92386$

$-0.68382$

0.60258

1.76112

0.98829

1.73922

1.74308

$-0.49675$

$-0.96038$

0.20392

$-0.48027$

$-0.94645$

0.21410

1.76249

0.60340

$-0.68588$

$-1.92552$

$\begin{array}{lrrr}\text { Re-F: } & & & \\ \text { Re } & 0.00000 & 0.00000 & 0.00000 \\ \text { F } & 0.00000 & 0.00000 & 1.90351 \\ \mathrm{O} & -1.34259 & 0.00088 & -3.84246 \\ \mathrm{O} & -0.08187 & 0.00068 & -2.00306 \\ \mathrm{~N} & -0.07356 & -1.94088 & -0.24832 \\ \mathrm{~N} & 2.02468 & 0.00000 & -0.39704 \\ \mathrm{~N} & -2.07590 & 0.00265 & -0.29205 \\ \mathrm{~N} & -0.07270 & 1.94110 & -0.24497 \\ \mathrm{C} & -2.44795 & 0.00519 & -1.73526 \\ \mathrm{C} & -1.23354 & 0.00182 & -2.65728 \\ \mathrm{C} & 2.14294 & -1.34788 & -1.16381 \\ \mathrm{C} & 0.99110 & -2.38431 & -0.92198 \\ \mathrm{C} & 2.14296 & 1.34894 & -1.16202 \\ \mathrm{C} & -1.33410 & -2.40952 & -0.23408 \\ \mathrm{C} & -2.35925 & -1.37963 & 0.33237 \\ \mathrm{C} & 0.99351 & 2.38667 & -0.91472 \\ \mathrm{C} & -2.35818 & 1.38278 & 0.33697 \\ \mathrm{C} & -1.33149 & 2.41345 & -0.22526 \\ \mathrm{C} & 2.94269 & -0.00057 & 0.76950 \\ \mathrm{C} & 0.85419 & -3.64244 & -1.48660 \\ \mathrm{C} & -1.51903 & -3.64566 & -0.81471 \\ \mathrm{C} & 0.85993 & 3.64939 & -1.46972 \\ \mathrm{C} & -1.51304 & 3.65483 & -0.79583 \\ \mathrm{C} & -0.39475 & -4.26210 & -1.38690 \\ \mathrm{C} & -0.38735 & 4.27180 & -1.36453 \\ \mathrm{H} & -3.06037 & -0.86660 & -1.96549 \\ \mathrm{H} & -3.05326 & 0.88236 & -1.96395 \\ \mathrm{H} & 2.12450 & 1.12903 & -2.22917 \\ \mathrm{H} & 3.10979 & 1.78679 & -0.91089 \\ \mathrm{H} & -3.38058 & -1.69260 & 0.11147 \\ \mathrm{H} & -2.24122 & -1.29526 & 1.41551\end{array}$




$\begin{array}{rrrr}\mathrm{H} & 3.97742 & -0.00007 & 0.41823 \\ \mathrm{H} & 2.75740 & -0.88628 & 1.37568 \\ \mathrm{H} & 2.75697 & 0.88423 & 1.37690 \\ \mathrm{H} & -0.51947 & -5.24053 & -1.83591 \\ \mathrm{H} & 1.65892 & -4.12271 & -2.02949 \\ \mathrm{H} & -2.48978 & -4.12328 & -0.86371 \\ \mathrm{H} & -0.50937 & 5.25407 & -1.80587 \\ \mathrm{H} & 1.66588 & 4.13181 & -2.00888 \\ \mathrm{H} & -2.48213 & 4.13637 & -0.83949 \\ \mathrm{H} & -2.24069 & 1.29474 & 1.41987 \\ \mathrm{H} & -3.37904 & 1.69767 & 0.11660 \\ \mathrm{H} & 3.10837 & -1.78756 & -0.91057 \\ \mathrm{H} & 2.12809 & -1.12631 & -2.23066\end{array}$

\begin{tabular}{|c|c|c|c|}
\hline $\mathrm{Re}$ & 0.00000 & 0.00000 & 0.00000 \\
\hline 0 & 0.00000 & 0.00000 & 1.70747 \\
\hline 0 & -1.15170 & 0.54624 & -4.07998 \\
\hline 0 & 0.01098 & 0.03764 & -2.23857 \\
\hline $\mathrm{N}$ & -0.09364 & -1.88416 & -0.53799 \\
\hline $\mathrm{N}$ & 1.95834 & 0.00007 & -0.68122 \\
\hline $\mathrm{N}$ & -2.02775 & -0.00103 & -0.62219 \\
\hline $\mathrm{N}$ & -0.09113 & 1.90663 & -0.51362 \\
\hline $\mathrm{C}$ & -2.31666 & 0.02096 & -2.07888 \\
\hline $\mathrm{C}$ & -1.06210 & 0.24689 & -2.91521 \\
\hline $\mathrm{C}$ & 2.09958 & -1.30064 & -1.48264 \\
\hline $\mathrm{C}$ & 0.93699 & -2.30011 & -1.30544 \\
\hline $\mathrm{C}$ & 2.12514 & 1.33607 & -1.41957 \\
\hline $\mathrm{C}$ & -1.36684 & -2.35531 & -0.64569 \\
\hline $\mathrm{C}$ & -2.39922 & -1.37463 & -0.06368 \\
\hline $\mathrm{C}$ & 0.93154 & 2.30728 & -1.29363 \\
\hline C & -2.39216 & 1.36890 & -0.05068 \\
\hline $\mathrm{C}$ & -1.36921 & 2.35268 & -0.64541 \\
\hline $\mathrm{C}$ & 2.91197 & -0.04465 & 0.45459 \\
\hline $\mathrm{C}$ & 0.77335 & -3.46575 & -2.02452 \\
\hline $\mathrm{C}$ & -1.57699 & -3.49310 & -1.38557 \\
\hline $\mathrm{C}$ & 0.75143 & 3.43832 & -2.06566 \\
\hline $\mathrm{C}$ & -1.59760 & 3.45455 & -1.43355 \\
\hline $\mathrm{C}$ & -0.47640 & -4.08903 & -2.01696 \\
\hline $\mathrm{C}$ & -0.50585 & 4.03931 & -2.08749 \\
\hline $\mathrm{H}$ & -2.73448 & -0.93900 & -2.38120 \\
\hline $\mathrm{H}$ & -3.06273 & 0.78300 & -2.29814 \\
\hline $\mathrm{H}$ & 2.24418 & 1.12830 & -2.48101 \\
\hline $\mathrm{H}$ & 3.04301 & 1.80271 & -1.05659 \\
\hline $\mathrm{H}$ & -3.41326 & -1.65618 & -0.35625 \\
\hline $\mathrm{H}$ & -2.33878 & -1.33482 & 1.02585 \\
\hline $\mathrm{H}$ & 3.93367 & -0.04037 & 0.06475 \\
\hline $\mathrm{H}$ & 2.74243 & -0.95138 & 1.03238 \\
\hline $\mathrm{H}$ & 2.75687 & 0.81910 & 1.09806 \\
\hline $\mathrm{H}$ & -0.61882 & -4.99887 & -2.58499 \\
\hline $\mathrm{H}$ & 1.57613 & -3.86482 & -2.63233 \\
\hline $\mathrm{H}$ & -2.56971 & -3.90800 & -1.50894 \\
\hline $\mathrm{H}$ & -0.66340 & 4.91682 & -2.70089 \\
\hline $\mathrm{H}$ & 1.54775 & 3.82069 & -2.69190 \\
\hline $\mathrm{H}$ & -2.59732 & 3.84225 & -1.58407 \\
\hline $\mathrm{H}$ & -2.31413 & 1.32515 & 1.03738 \\
\hline $\mathrm{H}$ & -3.41082 & 1.65022 & -0.32609 \\
\hline $\mathrm{H}$ & 3.05521 & -1.75947 & -1.22127 \\
\hline $\mathrm{H}$ & 2.11849 & -1.03894 & -2.53825 \\
\hline
\end{tabular}

$\begin{array}{llll}\text { Os-F: } & & & \\ \text { Os } & 0.00000 & 0.00000 & 0.00000 \\ \text { F } & 0.00000 & 0.00000 & 1.87389\end{array}$




$\begin{array}{lr}\mathrm{O} & -1.30893 \\ \mathrm{O} & -0.06704 \\ \mathrm{~N} & -0.04944 \\ \mathrm{~N} & 2.00261 \\ \mathrm{~N} & -2.05110 \\ \mathrm{~N} & -0.04837 \\ \mathrm{C} & -2.43221 \\ \mathrm{C} & -1.22095 \\ \mathrm{C} & 2.16830 \\ \mathrm{C} & 1.03261 \\ \mathrm{C} & 2.16900 \\ \mathrm{C} & -1.29806 \\ \mathrm{C} & -2.33919 \\ \mathrm{C} & 1.03544 \\ \mathrm{C} & -2.33772 \\ \mathrm{C} & -1.29536 \\ \mathrm{C} & 2.85216 \\ \mathrm{C} & 0.91944 \\ \mathrm{C} & -1.45787 \\ \mathrm{C} & 0.92572 \\ \mathrm{C} & -1.45166 \\ \mathrm{C} & -0.32037 \\ \mathrm{C} & -0.31260 \\ \mathrm{H} & -3.03493 \\ \mathrm{H} & -3.04410 \\ \mathrm{H} & 2.14512 \\ \mathrm{H} & 3.14590 \\ \mathrm{H} & -3.35593 \\ \mathrm{H} & -2.23830 \\ \mathrm{H} & 3.90378 \\ \mathrm{H} & 2.63549 \\ \mathrm{H} & 2.63603 \\ \mathrm{H} & -0.42581 \\ \mathrm{H} & 1.73529 \\ \mathrm{H} & -2.41586 \\ \mathrm{H} & -0.41526 \\ \mathrm{H} & 1.74309 \\ \mathrm{H} & -2.40792 \\ \mathrm{H} & -2.23698 \\ \mathrm{H} & -3.35409 \\ \mathrm{H} & 3.14424 \\ \mathrm{H} & 2.14639 \\ & \end{array}$

$\begin{array}{rr}0.02034 & -3.81096 \\ -0.00022 & -1.96123 \\ -1.91693 & -0.23190 \\ 0.00001 & -0.37817 \\ 0.00202 & -0.26910 \\ 1.91687 & -0.22913 \\ 0.00496 & -1.70671 \\ 0.01052 & -2.62675 \\ -1.34640 & -1.12391 \\ -2.38819 & -0.85646 \\ 1.34811 & -1.12102 \\ -2.41165 & -0.18407 \\ -1.38090 & 0.35260 \\ 2.39073 & -0.84856 \\ 1.38309 & 0.35698 \\ 2.41459 & -0.17559 \\ -0.00147 & 0.83984 \\ -3.67935 & -1.34838 \\ -3.68757 & -0.68061 \\ 3.68651 & -1.32899 \\ 3.69550 & -0.66050 \\ -4.31210 & -1.21766 \\ 4.32129 & -1.19259 \\ -0.87291 & -1.93998 \\ 0.87767 & -1.93489 \\ 1.14446 & -2.19200 \\ 1.76480 & -0.87159 \\ -1.70169 & 0.12067 \\ -1.28828 & 1.43629 \\ -0.00155 & 0.54318 \\ -0.88818 & 1.43397 \\ 0.88419 & 1.43571 \\ -5.31768 & -1.60826 \\ -4.17980 & -1.85502 \\ -4.19234 & -0.69294 \\ 5.33077 & -1.57383 \\ 4.18973 & -1.83039 \\ 4.20367 & -0.66686 \\ 1.28685 & 1.44037 \\ 1.70573 & 0.12605 \\ -1.76500 & -0.87391 \\ -1.14013 & -2.19441\end{array}$

$\begin{array}{lrrr}\text { Os-O: } & & & \\ \text { Os } & 0.00000 & 0.00000 & 0.00000 \\ \text { O } & 0.00000 & 0.00000 & 1.78226 \\ \text { O } & -1.37154 & -0.00236 & -3.92516 \\ \text { O } & -0.05376 & -0.00002 & -2.12321 \\ \text { N } & -0.07536 & -1.90460 & -0.33404 \\ \text { N } & 2.01035 & -0.00000 & -0.44454 \\ \text { N } & -2.05823 & 0.00123 & -0.34769 \\ \text { N } & -0.07462 & 1.90469 & -0.33224 \\ \text { C } & -2.40252 & 0.00261 & -1.79708 \\ \text { C } & -1.19393 & -0.00045 & -2.73352 \\ \text { C } & 2.13645 & -1.32260 & -1.22368 \\ \text { C } & 0.97837 & -2.34497 & -1.02282 \\ \text { C } & 2.13625 & 1.32282 & -1.22341 \\ \text { C } & -1.33342 & -2.37979 & -0.34721 \\ \text { C } & -2.36146 & -1.37244 & 0.24078 \\ \text { C } & 0.97970 & 2.34626 & -1.01932 \\ \text { C } & -2.36069 & 1.37388 & 0.24340 \\ \text { C } & -1.33183 & 2.38181 & -0.34221 \\ \text { C } & 2.95185 & 0.00004 & 0.69038\end{array}$




$\begin{array}{lr}\mathrm{C} & 0.83044 \\ \mathrm{C} & -1.52919 \\ \mathrm{C} & 0.83317 \\ \mathrm{C} & -1.52609 \\ \mathrm{C} & -0.41822 \\ \mathrm{C} & -0.41459 \\ \mathrm{H} & -3.01355 \\ \mathrm{H} & -3.00773 \\ \mathrm{H} & 2.12539 \\ \mathrm{H} & 3.09858 \\ \mathrm{H} & -3.38056 \\ \mathrm{H} & -2.23486 \\ \mathrm{H} & 3.98217 \\ \mathrm{H} & 2.77327 \\ \mathrm{H} & 2.77355 \\ \mathrm{H} & -0.55250 \\ \mathrm{H} & 1.63401 \\ \mathrm{H} & -2.50801 \\ \mathrm{H} & -0.54766 \\ \mathrm{H} & 1.63707 \\ \mathrm{H} & -2.50405 \\ \mathrm{H} & -2.23439 \\ \mathrm{H} & -3.37949 \\ \mathrm{H} & 3.09771 \\ \mathrm{H} & 2.12851\end{array}$

-3.57184
-3.58566
3.57580
3.59090
-4.18918
4.19482
-0.86896
0.87837
1.08126
1.77830
-1.69079
-1.30949
-0.00017
-0.88194
0.88227
-5.13962
-4.02316
-4.04217
5.14762
4.02843
4.04979
1.30877
1.69344
-1.77920
-1.08070

Ir-F:

$\begin{array}{lr}\text { Ir } & 0.00000 \\ \mathrm{~F} & 0.00000 \\ \mathrm{O} & -1.49691 \\ \mathrm{O} & -0.13851 \\ \mathrm{~N} & -0.02952 \\ \mathrm{~N} & 1.97536 \\ \mathrm{~N} & -2.02464 \\ \mathrm{~N} & -0.02935 \\ \mathrm{C} & -2.48682 \\ \mathrm{C} & -1.32435 \\ \mathrm{C} & 2.15513 \\ \mathrm{C} & 1.04959 \\ \mathrm{C} & 2.15536 \\ \mathrm{C} & -1.25707 \\ \mathrm{C} & -2.28683 \\ \mathrm{C} & 1.05081 \\ \mathrm{C} & -2.28646 \\ \mathrm{C} & -1.25615 \\ \mathrm{C} & 2.78071 \\ \mathrm{C} & 0.95701 \\ \mathrm{C} & -1.40269 \\ \mathrm{C} & 0.96010 \\ \mathrm{C} & -1.40010 \\ \mathrm{C} & -0.26759 \\ \mathrm{C} & -0.26398 \\ \mathrm{H} & -3.10259 \\ \mathrm{H} & -3.09944 \\ \mathrm{H} & 2.08465 \\ \mathrm{H} & 3.15118 \\ \mathrm{H} & -3.31026 \\ \mathrm{H} & -2.15213 \\ \mathrm{H} & 3.83982 \\ \mathrm{H} & 2.54241 \\ \mathrm{H} & 2.54313 \\ \mathrm{H} & 1.77870 \\ \mathrm{H} & \\ & \end{array}$

$-1.65170$

$-0.98697$

$-1.64321$

$-0.97637$

$-1.59057$

$-1.57853$

$-2.03093$

$-2.03047$

$-2.28568$

$-0.98188$

0.00686

1. 32310

0.32067

1.30238

1. 30212

$-2.09181$

$-2.22004$

$-1.06174$

$-2.07561$

$-2.21006$

$-1.04784$

1. 32562

0.00977

$-0.98008$

$-2.28591$

0.00000

1.89951

$-3.72393$

$-1.95243$

$-0.13664$

$-0.34225$

$-0.14573$

$-0.13503$

$-1.55716$

$-2.55021$

$-1.07559$

$-0.73615$

$-1.07362$

$-0.00796$

0.51666

$-0.73135$

0.51873

$-0.00388$

0.90979

$-1.12339$

$-0.40825$

$-1.11245$

$-0.39872$

$-0.92468$

$-0.91172$

$-1.75479$

$-1.75403$

$-2.14446$

$-0.85299$

0.31860

1.59225

0.64286

1. 49492

1.49625

$-1.23421$

$-1.59655$ 


$\begin{array}{rr}\mathrm{H} & -2.34877 \\ \mathrm{H} & -0.35422 \\ \mathrm{H} & 1.78292 \\ \mathrm{H} & -2.34542 \\ \mathrm{H} & -2.15212 \\ \mathrm{H} & -3.30973 \\ \mathrm{H} & 3.15049 \\ \mathrm{H} & 2.08571\end{array}$

$$
\begin{array}{r}
-4.25918 \\
5.41604 \\
4.25990 \\
4.26295 \\
1.24663 \\
1.69894 \\
-1.73927 \\
-1.15623
\end{array}
$$

$$
\begin{array}{r}
-0.34999 \\
-1.21630 \\
-1.58198 \\
-0.33788 \\
1.59416 \\
0.32084 \\
-0.85447 \\
-2.14619
\end{array}
$$

$$
\text { Ir-O: }
$$

$$
\text { Ir }
$$

$$
0.00000
$$

0.00000

0.00000

0.00860

$-1.51751$

$-0.08298$

$-0.04145$

0.00080

$-1.88991$

2.00466

$-2.01877$

$-0.02491$

$-2.42765$

$-1.26547$

2.17349

1.05907

2.18529

$-1.26948$

$-2.30600$

1.08090

$-2.29405$

$-1.24789$

2.80534

0.97453

$-1.40437$

1.00952

$-1.36993$

$-0.25939$

$-0.21850$

$-3.04748$

$-3.03905$

2.10777

3.17973

$-3.32525$

$-2.17294$

3.87133

2.54997

2.55447

$-0.34590$

1.81082

$-2.35343$

$-0.29475$

1.85146

$-2.31384$

$-2.16303$

$-3.31020$

3.16409

2.09866

$-0.00840$

0.00924

1.89022

0.01248

0.00698

$-1.34190$

$-2.39744$

1. 32542

$-2.40271$

$-1.35162$

2. 39024

1. 37127

2. 41424

$-0.01340$

$-3.72624$

$-3.71874$

3. 72196

3. 73374

$-4.36491$

4.37153

$-0.86019$

0.89134

1.10851

1.72410

$-1.67935$

$-1.23055$

$-0.01578$

$-0.89540$

0.86710

$-5.39784$

$-4.25305$

$-4.23844$

5.40710

4.24349

4.26256

1. 24667

1.70863

$-1.75024$

$-1.12143$

$$
0.00000
$$

1.88750

$-3.78585$

$-2.07687$

$-0.14014$

$-0.22992$

$-0.17172$

$-0.13703$

$-1.60638$

$-2.60878$

$-0.98163$

$-0.68499$

$-0.97823$

$-0.03181$

0.47016

$-0.67791$

0.47285

$-0.02601$

1.00628

$-1.07118$

$-0.43418$

$-1.05644$

$-0.42145$

$-0.91647$

$-0.89925$

$-1.81121$

$-1.80990$

$-2.04412$

$-0.76809$

0.25580

1.54691

0.76015

1.59172

1.59588

$-1.23074$

$-1.51278$

$-0.40268$

$-1.20747$

$-1.49353$

$-0.38698$

1. 54948

0.25868

$-0.77198$

$-2.04697$

$\begin{array}{lrrr}\text { Pt-F: } & & & \\ \text { Pt } & 0.00000 & 0.00000 & 0.00000 \\ \text { F } & 0.00000 & 0.00000 & 1.94558 \\ \text { O } & -1.57001 & -0.00215 & -3.62722 \\ \text { O } & -0.09393 & -0.00007 & -1.97140 \\ \text { N } & 0.00018 & -1.88910 & -0.00945 \\ \text { N } & 2.00164 & -0.00000 & -0.04838 \\ \text { N } & -1.99159 & -0.00011 & -0.00927\end{array}$




$\begin{array}{lr}\text { N } & -0.00026 \\ \mathrm{C} & -2.46172 \\ \mathrm{C} & -1.33377 \\ \mathrm{C} & 2.23996 \\ \mathrm{C} & 1.17362 \\ \mathrm{C} & 2.24003 \\ \mathrm{C} & -1.18304 \\ \mathrm{C} & -2.24929 \\ \mathrm{C} & 1.17354 \\ \mathrm{C} & -2.24974 \\ \mathrm{C} & -1.18331 \\ \mathrm{C} & 2.66465 \\ \mathrm{C} & 1.19339 \\ \mathrm{C} & -1.21243 \\ \mathrm{C} & 1.19360 \\ \mathrm{C} & -1.21241 \\ \mathrm{C} & -0.01012 \\ \mathrm{C} & -0.00990 \\ \mathrm{H} & -3.08467 \\ \mathrm{H} & -3.08167 \\ \mathrm{H} & 2.14063 \\ \mathrm{H} & 3.24987 \\ \mathrm{H} & -3.26174 \\ \mathrm{H} & -2.14549 \\ \mathrm{H} & 3.74561 \\ \mathrm{H} & 2.36644 \\ \mathrm{H} & 2.36649 \\ \mathrm{H} & -0.01286 \\ \mathrm{H} & 2.08747 \\ \mathrm{H} & -2.11194 \\ \mathrm{H} & -0.01240 \\ \mathrm{H} & 2.08793 \\ \mathrm{H} & -2.11181 \\ \mathrm{H} & -2.14673 \\ \mathrm{H} & -3.26207 \\ \mathrm{H} & 3.24990 \\ \mathrm{H} & 2.14016\end{array}$

$\begin{array}{rr}1.88907 & -0.00678 \\ 0.00083 & -1.42738 \\ -0.00071 & -2.46329 \\ -1.35842 & -0.74773 \\ -2.42178 & -0.33483 \\ 1.36003 & -0.74465 \\ -2.42445 & 0.27745 \\ -1.35875 & 0.68454 \\ 2.42245 & -0.32976 \\ 1.35733 & 0.68648 \\ 2.42381 & 0.28181 \\ -0.00176 & 1.27536 \\ -3.80766 & -0.33690 \\ -3.80889 & 0.24008 \\ 3.80834 & -0.32779 \\ 3.80835 & 0.24846 \\ -4.46671 & -0.05381 \\ 4.46679 & -0.04328 \\ -0.87513 & -1.60790 \\ 0.87899 & -1.60757 \\ 1.20199 & -1.82075 \\ 1.70757 & -0.52467 \\ -1.70033 & 0.46988 \\ -1.18886 & 1.75815 \\ -0.00165 & 1.11917 \\ -0.88496 & 1.83639 \\ 0.87990 & 1.83877 \\ -5.55092 & -0.06798 \\ -4.37411 & -0.56431 \\ -4.37623 & 0.44233 \\ 5.55104 & -0.05406 \\ 4.37527 & -0.55303 \\ 4.37528 & 0.45234 \\ 1.18581 & 1.75988 \\ 1.69914 & 0.47159 \\ -1.70633 & -0.52884 \\ -1.19805 & -1.82343\end{array}$

$\begin{array}{lrrr}\text { Pt-O: } & & & \\ \text { Pt } & 0.00000 & 0.00000 & 0.00000 \\ \text { O } & 0.00000 & 0.00000 & 1.92128 \\ \text { O } & -1.85435 & -0.01643 & -3.62276 \\ \text { O } & -0.20149 & -0.00192 & -2.12350 \\ \text { N } & -0.00032 & -1.88155 & -0.01863 \\ \text { N } & 1.99990 & -0.00000 & -0.08141 \\ \text { N } & -1.98486 & 0.00014 & 0.02676 \\ \text { N } & -0.00094 & 1.88147 & -0.01623 \\ \text { C } & -2.50825 & 0.00186 & -1.36722 \\ \text { C } & -1.44343 & -0.00725 & -2.49175 \\ \text { C } & 2.21014 & -1.33974 & -0.80367 \\ \text { C } & 1.15460 & -2.41254 & -0.38979 \\ \text { C } & 2.20995 & 1.34125 & -0.80095 \\ \text { C } & -1.18190 & -2.41189 & 0.27547 \\ \text { C } & -2.20035 & -1.34033 & 0.74985 \\ \text { C } & 1.15464 & 2.41325 & -0.38442 \\ \text { C } & -2.20033 & 1.33885 & 0.75326 \\ \text { C } & -1.18198 & 2.41137 & 0.28067 \\ \text { C } & 2.68324 & -0.00117 & 1.22461 \\ \text { C } & 1.16068 & -3.79724 & -0.44920 \\ \text { C } & -1.22736 & -3.79499 & 0.18341 \\ \text { C } & 1.16155 & 3.79817 & -0.43845 \\ \text { C } & -1.22664 & 3.79486 & 0.19361 \\ \text { C } & -0.04310 & -4.45414 & -0.16479\end{array}$




$\begin{array}{rrrr}\mathrm{C} & -0.04203 & 4.45466 & -0.15221 \\ \mathrm{H} & -3.14717 & -0.86899 & -1.51233 \\ \mathrm{H} & -3.13075 & 0.88416 & -1.51496 \\ \mathrm{H} & 2.06402 & 1.16240 & -1.86913 \\ \mathrm{H} & 3.22561 & 1.70004 & -0.62641 \\ \mathrm{H} & -3.23271 & -1.67560 & 0.64956 \\ \mathrm{H} & -1.92196 & -1.13159 & 1.79085 \\ \mathrm{H} & 3.76382 & 0.00026 & 1.05443 \\ \mathrm{H} & 2.36616 & -0.87305 & 1.79192 \\ \mathrm{H} & 2.36409 & 0.86825 & 1.79452 \\ \mathrm{H} & -0.06048 & -5.53597 & -0.22744 \\ \mathrm{H} & 2.04155 & -4.36187 & -0.72495 \\ \mathrm{H} & -2.13009 & -4.35777 & 0.38150 \\ \mathrm{H} & -0.05879 & 5.53673 & -0.21071 \\ \mathrm{H} & 2.04292 & 4.36334 & -0.71147 \\ \mathrm{H} & -2.12900 & 4.35739 & 0.39405 \\ \mathrm{H} & -1.92097 & 1.12773 & 1.79356 \\ \mathrm{H} & -3.23280 & 1.67413 & 0.65422 \\ \mathrm{H} & 3.22574 & -1.69889 & -0.62952 \\ \mathrm{H} & 2.06449 & -1.15878 & -1.87151\end{array}$

\subsection{Force Constants}

Table S2: Force constants of $\mathrm{M}-\mathrm{X}$ bonds.

\begin{tabular}{|c|c|c|}
\hline $\begin{array}{l}\text { Metal } \\
\text { Center }\end{array}$ & $\begin{array}{c}\text { M-F Force } \\
\text { Constant } \\
\text { [mdyn/Å] }\end{array}$ & $\begin{array}{c}\text { M-O Force } \\
\text { Constant } \\
\text { [mdyn/Å] }\end{array}$ \\
\hline $\mathrm{Mn}$ & 3.1554 & 5.5674 \\
\hline Tc & 3.7296 & 7.4058 \\
\hline $\operatorname{Re}$ & 3.9236 & 7.8511 \\
\hline $\mathrm{Fe}$ & 3.1594 & 5.6370 \\
\hline $\mathrm{Ru}$ & 3.9444 & 5.5056 \\
\hline Os & 4.2177 & 5.9290 \\
\hline Co & 2.6573 & 1.9402 \\
\hline $\mathrm{Rh}$ & 2.8038 & 2.3434 \\
\hline Ir & 3.9608 & 2.9606 \\
\hline $\mathrm{Ni}$ & 2.7342 & 2.1208 \\
\hline $\mathrm{Pd}$ & 2.9803 & 3.1830 \\
\hline $\mathrm{Pt}$ & 3.3245 & 3.6240 \\
\hline
\end{tabular}

\section{AILFT Analysis}

\subsection{Methodology}

The sophisticated geometry of the complexes lead to the following method to derive the AOM parameters:

- For every of the 12 metals, a geometry optimization of a $\mathrm{M}\left(\mathrm{NH}_{3}\right)_{6}{ }^{4+}$ complex was performed. 
- The $\mathrm{X}$ ligand of every of the $24 \mathrm{M}-\mathrm{X}$ complex was substituted by a $\mathrm{NH}_{3}$ molecule with the $\mathrm{NH}_{3}$ geometry as well as the $\mathrm{M}-\mathrm{N}$ bond length derived from the $\mathrm{M}\left(\mathrm{NH}_{3}\right)_{6}{ }^{4+}$ calculation.

- For every complex - the original $\mathrm{M}-\mathrm{X}$, the newly designed $\mathrm{M}-\mathrm{NH}_{3}$ as well as the $\mathrm{M}\left(\mathrm{NH}_{3}\right)_{6}{ }^{4+}-$ an AILFT calculation was performed (total of 60).

- From the $\mathrm{M}\left(\mathrm{NH}_{3}\right)_{6}{ }^{4+}$ AILFT calculations, $\mathrm{e}_{\sigma}\left(\mathrm{NH}_{3}\right)$ was derived for every $\mathrm{M}$ by standard procedure $\left(\frac{10 \mathrm{Dq}}{3}\right)$ and $\mathrm{e}_{\pi}\left(\mathrm{NH}_{3}\right)$ was approximated to be zero.

- $\mathrm{e}_{\sigma}, \mathrm{e}_{\pi \mathrm{s}}$ and $\mathrm{e}_{\pi \mathrm{c}}$ for $\mathrm{X}=\mathrm{F}$ and $\mathrm{X}=\mathrm{O}$ were calculated for every $\mathrm{M}$ the following way:

$$
\begin{gathered}
e_{\pi s}(\mathrm{X}) \approx d_{y z}(\mathrm{X})-d_{y z}\left(\mathrm{NH}_{3}\right) ; e_{\pi c}(\mathrm{X}) \approx d_{x z}(\mathrm{X})-d_{x z}\left(\mathrm{NH}_{3}\right) \\
e_{\sigma}(\mathrm{X}) \approx\left(d_{x^{2}-y^{2}}+d_{z^{2}}\right)_{\mathrm{X}}-\left(d_{x^{2}-y^{2}}+d_{z^{2}}\right)_{\mathrm{NH}_{3}}+e_{\sigma}\left(\mathrm{NH}_{3}\right)
\end{gathered}
$$

\subsection{Mn Model Complex}

As an example, the AILFT output of the $\mathrm{Mn}-\mathrm{X}$ complexes as well as pictures of the resulting $d$ orbitals (iso $=0.05$ ) are given here. It can be seen (in the numbers as well as in the pictures) that the $d$ orbitals with $t_{2 g}$ symmetry are fairly pure, while the orbitals with $e_{g}$ symmetry are mixed. This mixing was found to be independent from the orientation in space, i.e. how the axes were defined.

$\mathrm{Mn}-\mathrm{F}$ :

\begin{tabular}{|c|c|c|c|c|}
\hline 0 & & $=$ & . & a.u. \\
\hline (dxy & dxy & )$=$ & -0.024300978 & a.u. \\
\hline$y z$ & dxy & $=$ & 0.005051520 & a.u. \\
\hline$y z$ & dyz & $=$ & 7699112 & a.u. \\
\hline 22 & dxy & $=$ & 42 & a.u. \\
\hline & dyz & )$=$ & 205 & a.u. \\
\hline$(\mathrm{dz} 2$ &, $\mathrm{dz} 2$ & )$=$ & 0 . & a.u. \\
\hline & dxy & $=$ & -0 . & a.u. \\
\hline$z$ & dyz & )$=$ & -0 . & a.u. \\
\hline$z$ & $\mathrm{dz} 2$ & )$=$ & 360 & a.u. \\
\hline$(d x z$ & $\mathrm{dxz}$ & $=$ & 105029 & a.u. \\
\hline$\left(d x 2-y^{2}\right.$ & $x y$ & )$=$ & 7717 & a.u. \\
\hline $2-y^{2}$ & $\mathrm{z}$ & )$=$ & -0 . & a.u. \\
\hline$\left(d x 2-y^{2}\right.$ & & )$=$ & 186694 & a.u. \\
\hline$\left(d \times 2-y^{2}\right.$ & $x z$ & )$=$ & 0.000048309 & a.u. \\
\hline & & 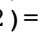 & 0.1342 & a.u. \\
\hline & & $=$ & 0.0043 & a.u. \\
\hline
\end{tabular}

AILFT MATRIX ELEMENTS (CASSCF)

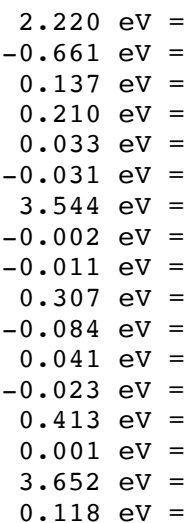

$17904.2 \mathrm{Cm} * *-1$

$-5333.4 \mathrm{~cm} * *-1$

$1108.7 \mathrm{Cm} * *-1$

$1689.8 \mathrm{~cm} * *-1$

$267.9 \mathrm{~cm} * *-1$

$-248.1 \mathrm{~cm} * *-1$

$28581.4 \mathrm{Cm} * *-1$

$-18.6 \mathrm{~cm} * *-1$

$-88.7 \mathrm{Cm} * *-1$

$2478.4 \mathrm{~cm} * *-1$

$-681.5 \mathrm{~cm} * *-1$

$330.9 \mathrm{~cm} * *-1$

$-185.5 \mathrm{~cm} * *-1$

$3333.1 \mathrm{~cm} * *-1$

$10.6 \mathrm{Cm} * *-1$

$29456.3 \mathrm{~cm} * *-1$

$955.0 \mathrm{~cm} * *-1$

\begin{tabular}{|c|c|c|c|c|c|c|c|}
\hline rbital & Energy (eV) & Energy $(\mathrm{cm}-1)$ & $d x y$ & dyz & $\mathrm{dz} 2$ & $d x z$ & $\mathrm{dx} 2-\mathrm{y}$ \\
\hline 3 & 0.914 & 7370.7 & -0.152834 & -0.987852 & -0.009610 & 0.026183 & -0.00 \\
\hline
\end{tabular}

The ligand field one electron eigenfunctions:

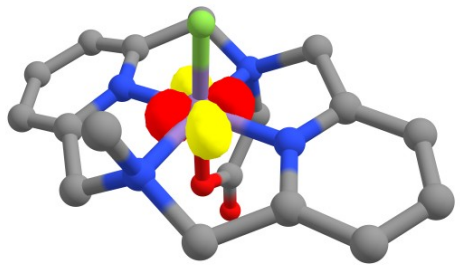

3:

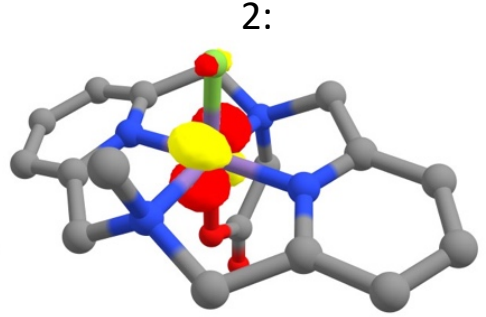

4: 


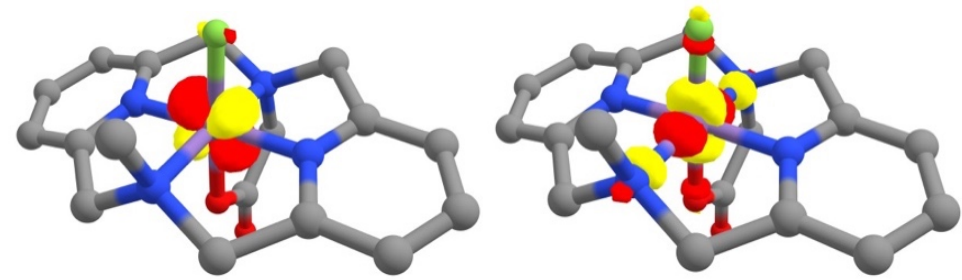

5:

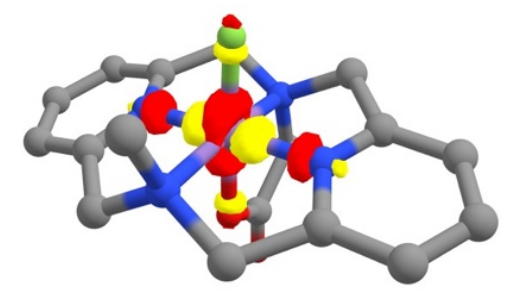

$\mathrm{M}-\mathrm{O}$ :

AILFT MATRIX ELEMENTS (CASSCF)

\begin{tabular}{|c|c|c|c|c|c|c|c|c|c|}
\hline E0 & & $=$ & 0.068197099 & a.u. $=$ & 1.856 & $\mathrm{eV}$ & $=$ & 14967.5 & $\mathrm{~cm} * *-1$ \\
\hline $\mathrm{H}(\mathrm{dxy}$ & , dxy & )$=$ & -0.048926245 & a.u. $=$ & -1.331 & $\mathrm{eV}$ & $=$ & -10738.1 & $\mathrm{~cm} * *-1$ \\
\hline $\mathrm{H}(\mathrm{dyz}$ & dxy & )$=$ & 0.003389399 & $\mathrm{a} \cdot \mathrm{u} \cdot=$ & 0.092 & $\mathrm{eV}$ & $=$ & 743.9 & $\mathrm{~cm} * *-1$ \\
\hline $\mathrm{H}(\mathrm{dyz}$ & dyz & )$=$ & 0.025619161 & a.u. $=$ & 0.697 & $\mathrm{eV}$ & $=$ & 5622.8 & $\mathrm{~cm} * *-1$ \\
\hline $\mathrm{H}(\mathrm{dz} 2$ & dxy & )$=$ & 0.000666761 & a.u. $=$ & 0.018 & $\mathrm{eV}$ & $=$ & 146.3 & $\mathrm{~cm} * *-1$ \\
\hline $\mathrm{H}(\mathrm{dz} 2$ & dyz & )$=$ & 0.000076175 & $\mathrm{a} \cdot \mathrm{u} \cdot=$ & 0.002 & $\mathrm{eV}$ & $=$ & 16.7 & $\mathrm{~cm} * *-1$ \\
\hline $\mathrm{H}(\mathrm{dz} 2$ & $\mathrm{dz} 2$ & )$=$ & 0.127291360 & a.u. $=$ & 3.464 & $\mathrm{eV}$ & $=$ & 27937.2 & $\mathrm{~cm} * *-1$ \\
\hline$H(d x z$ & dxy & )$=$ & -0.000258662 & $\mathrm{a} \cdot \mathrm{u} \cdot=$ & -0.007 & $\mathrm{eV}$ & $=$ & -56.8 & $\mathrm{~cm} * *-1$ \\
\hline $\mathrm{H}(\mathrm{dxz}$ & dyz & )$=$ & 0.000034542 & a.u. = & 0.001 & $\mathrm{eV}$ & $=$ & 7.6 & $\mathrm{~cm} * *-1$ \\
\hline $\mathrm{H}(\mathrm{dxz}$ &, $\mathrm{dz} 2$ & )$=$ & 0.002063772 & a.u. $=$ & 0.056 & $\mathrm{eV}$ & $=$ & 452.9 & $\mathrm{~cm} * *-1$ \\
\hline$H(d x z$ & $\mathrm{dxz}$ & )$=$ & 0.022722861 & a.u. $=$ & 0.618 & $\mathrm{eV}$ & $=$ & 4987.1 & $\mathrm{~cm} * *-1$ \\
\hline $\mathrm{H}\left(\mathrm{dx} 2-\mathrm{y}^{2}\right.$ & dxy & )$=$ & 0.000224850 & a.u. = & 0.006 & $\mathrm{eV}$ & $=$ & 49.3 & $\mathrm{~cm} * *-1$ \\
\hline $\mathrm{H}\left(\mathrm{d} \times 2-\mathrm{y}^{2}\right.$ & , dyz & )$=$ & -0.000101887 & a.u. $=$ & -0.003 & $\mathrm{eV}$ & $=$ & -22.4 & $\mathrm{~cm} * *-1$ \\
\hline $\mathrm{H}\left(\mathrm{dx} 2-\mathrm{y}^{2}\right.$ &, $\mathrm{dz} 2$ & )$=$ & 0.016243286 & a.u. = & 0.442 & $\mathrm{eV}$ & $=$ & 3565.0 & $\mathrm{~cm} * *-1$ \\
\hline $\mathrm{H}(\mathrm{d} \times 2-\mathrm{y} 2$ &, $\mathrm{dxz}$ & )$=$ & 0.000234679 & $\mathrm{a} \cdot \mathrm{u} \cdot=$ & 0.006 & $\mathrm{eV}$ & $=$ & 51.5 & $\mathrm{~cm} * *-1$ \\
\hline $\mathrm{H}\left(\mathrm{dx} 2-\mathrm{y}^{2}\right.$ & ( & $2)=$ & 0.077884160 & $\mathrm{a} \cdot \mathrm{u} .=$ & 2.119 & $\mathrm{eV}$ & $=$ & 17093.6 & $\mathrm{~cm} * *-1$ \\
\hline B & & $=$ & 0.004484069 & a.u. $=$ & 0.122 & $\mathrm{eV}$ & $=$ & 984.1 & $\mathrm{~cm} * *-1$ \\
\hline
\end{tabular}

The ligand field one electron eigenfunctions:

\begin{tabular}{cccccrrr} 
rbital & Energy $(\mathrm{eV})$ & Energy $(\mathrm{cm}-1)$ & \multicolumn{1}{c}{$\mathrm{dxy}$} & \multicolumn{1}{c}{$\mathrm{dyz}$} & \multicolumn{1}{c}{$\mathrm{dz2}$} & $\mathrm{dxz}$ \\
1 & 0.000 & 0.0 & -0.998958 & 0.045324 & 0.003677 & -0.003730 & 0.001342 \\
2 & 1.953 & 15751.0 & 0.004098 & 0.006458 & 0.019945 & -0.999771 & -0.001623 \\
3 & 2.037 & 16429.1 & -0.045296 & -0.998948 & 0.001534 & -0.006604 & -0.002206 \\
4 & 3.323 & 26799.8 & 0.000146 & -0.002514 & -0.286492 & -0.007287 & 0.958052 \\
5 & 4.933 & 39785.9 & -0.003865 & -0.000540 & -0.957867 & -0.018663 & -0.286579
\end{tabular}

1:

2:

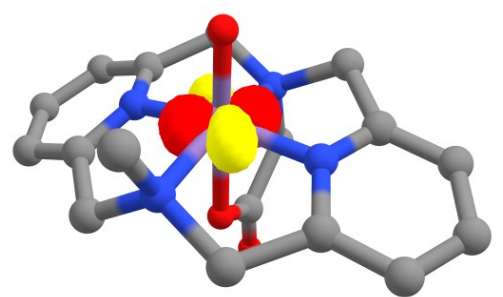

3:

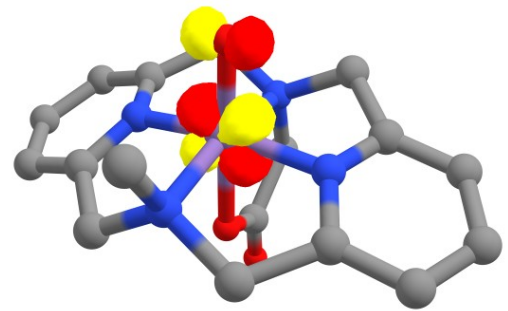

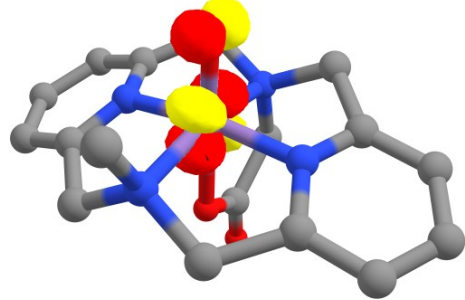

4:

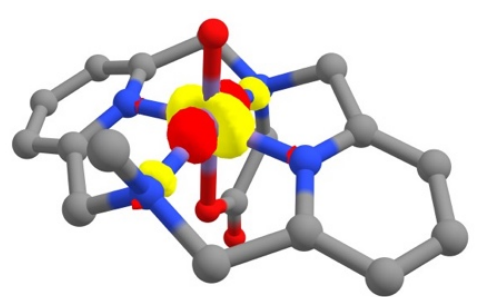

5: 


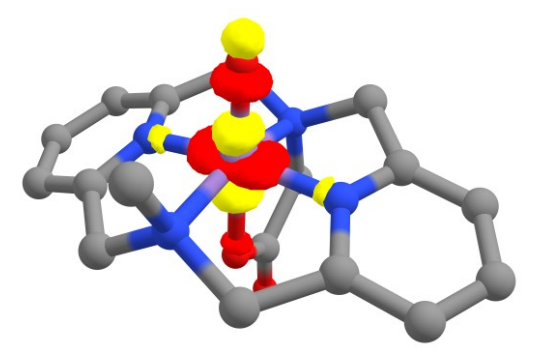

\subsection{Full AOM Analysis}

AOM Parameters were calculated for every complex except those with oxyl radical ligands, because for CASSCF calculations the inactive space has to be closed shell. For $\operatorname{Re}-\mathrm{O}$, the $d_{\mathrm{z}^{2}}$ orbital could not be found, so no $e_{\sigma}(\operatorname{Re}-0)$ is given.

Table S3: AOM parameters for every $\mathrm{M}-\mathrm{X}$ complex.

\begin{tabular}{l|crc|ccc}
$\mathrm{M}=$ & $\mathrm{e}_{\sigma}(\mathrm{F})$ & \multicolumn{1}{c}{$\mathrm{e}_{\pi s}(\mathrm{~F})$} & $\mathrm{e}_{\pi c}(\mathrm{~F})$ & $\mathrm{e}_{\sigma}(\mathrm{O})$ & $\mathrm{e}_{\pi s}(\mathrm{O})$ & $\mathrm{e}_{\pi c}(\mathrm{O})$ \\
\hline $\mathrm{Mn}$ & 10799.4 & 1201.5 & 2959.3 & 13454.4 & 10837.5 & 14644.4 \\
$\mathrm{Fe}$ & 11063.7 & 1155.3 & 2766.1 & 12196.4 & 9459.5 & 13124.7 \\
$\mathrm{Co}$ & 11350.8 & 369.7 & 2113.5 & - & - & - \\
$\mathrm{Ni}$ & 14050.6 & 48.8 & 2042.2 & - & - & - \\
$\mathrm{Tc}$ & 16332.6 & 3053.2 & 4788.8 & 25554.9 & 13883.1 & 15169.2 \\
$\mathrm{Ru}$ & 17199.4 & 2902.2 & 4756.8 & 21886.5 & 10640.6 & 15073.1 \\
$\mathrm{Rh}$ & 15609.4 & 615.3 & 2890.1 & - & - & - \\
$\mathrm{Pd}$ & 16771.3 & 1116.5 & 2749.5 & 16098.0 & 6738.0 & 9165.9 \\
$\mathrm{Re}$ & 19380.1 & 4103.3 & 5957.7 & - & 14983.7 & 15309.1 \\
$\mathrm{Os}$ & 19528.7 & 3736.8 & 5536.2 & 33430.7 & 9503.8 & 14554.5 \\
$\mathrm{Ir}$ & 17959.6 & 2516.1 & 4587.6 & - & - & - \\
$\mathrm{Pt}$ & 16633.3 & 3075.4 & 3075.4 & 20702.7 & 6962.7 & 13955.2 \\
\hline Mean & 15556.6 & 1991.2 & 3685.3 & 20357.3 & 10350.8 & 13875.6
\end{tabular}

